\title{
Compact sources in the Bologna Complete Sample: high-resolution VLA observations and optical data
}

\author{
E. Liuzzo ${ }^{1}$, S. Buttiglione ${ }^{2}$, G. Giovannini ${ }^{1,3}$, M. Giroletti ${ }^{1}$, A. Capetti ${ }^{4}$, and G. B. Taylor ${ }^{5, \star}$ \\ 1 INAF - Istituto di Radioastronomia, via Gobetti 101, 40129 Bologna, Italy \\ e-mail: liuzzo@ira.inaf.it \\ 2 INAF - Osservatorio Astronomico di Padova, vicolo dell Osservatorio 5, 35122 Padova, Italy \\ 3 Dipartimento di Astronomia, Universitá di Bologna, via Ranzani 1, 40127 Bologna, Italy \\ ${ }^{4}$ INAF - Osservatorio Astronomico di Torino, via Osservatorio 20, 10025 Pino Torinese, Italy \\ 5 Department of Physics and Astronomy, University of New Mexico, Albuquerque NM 87131, USA
}

Received 13 July 2012 / Accepted 18 September 2012

\begin{abstract}
Context. Among radio galaxies, compact sources are a class of objects that are not yet well understood, and most of them cannot be included in classical populations of compact radio sources (flat-spectrum or compact steep-spectrum sources).

Aims. Our main goal is to analyze the radio and optical properties of a sample of compact sources and compare them with Faranoff-Riley I and II extended radio galaxies.

Methods. We selected in the Bologna Complete Sample a subsample of compact sources, naming it the C BCS sample. We collected new and literature subarcsecond resolution multi-frequency VLA images and optical data. We compared total and nuclear radio power with optical emission line measurements.

Results. The [OIII] luminosity - $408 \mathrm{MHz}$ total power relation found in high- and low-excitation galaxies as well as in young (CSS) sources holds also for the C BCSs. However, C BCSs present higher [OIII] luminosity than expected at a given total radio power, and they show the same correlation as core radio galaxies, but with a higher radio power.

Conclusions. The C BCSs appear to be the high-power tail of core radio galaxies. For most of the C BCSs, the morphology seems to be strongly dependent on the presence of dense environments (e.g., cluster or HI-rich galaxies) and on a young age or restarted radio activity.
\end{abstract}

Key words. radio continuum: galaxies - galaxies: active - galaxies: jets

\section{Introduction}

According to their radio power and morphologies, radio galaxies are classified into FRI and FRII (Fanaroff \& Riley 1974) and compact sources. The FR I and FR II galaxies are extended sources on kpc up to Mpc scale. Their properties have been analyzed by several authors (e.g., Fanaroff \& Riley 1974; Laing et al. 1983; Fanti et al. 1986; Ledlow \& Owen 1996). Recently, Capetti et al. (2011, and references therein) discussed the correlation between optical and radio properties at the light of unification models and accretion properties.

Sources with a projected linear size smaller than $15-20 \mathrm{kpc}$ are usually defined as compact sources and can be high- or lowpower radio sources. High-radio power sources can have a flat or a steep spectrum. Flat-spectrum sources are small because of projection and relativistic effects being identified (in agreement with unified models, see e.g., Urry \& Padovani 1995) as objects dominated by the emission of a relativistic jet oriented at a small angle with respect to the line of sight. According to their radio power and optical properties, they are classified as flat spectrum radio quasars (FSRQ) or BL Lac objects.

\footnotetext{
* Adjunct Astronomer at the National Radio Astronomy Observatory, USA.
}

High-power compact steep spectrum (CSS) sources can be small because they are young sources (e.g., Stanghellini et al. 2005 , and references therein). They are not beamed sources and they are likely to be young radio galaxies that could evolve into large radio objects, FR I/FR II (see Fanti et al. 1995; Readhead et al. 1996; O’Dea 1998, for reviews, but also van Breugel et al. 1984). Strong support for this scenario comes from the measurements of proper motions of the hot spots of some of these galaxies (Polatidis \& Conway 2003; Giroletti \& Panessa 2009), with separation velocities of $0.1-0.4 \mathrm{~h}^{-1} \mathrm{c}$, and the young kinematic ages that agree with spectral ages derived from flux density measurements (Murgia et al. 1999; Murgia 2003). Kunert-Bajraszewska et al. (2010) selected and studied the properties of low-power CSS sources with flux density $<70 \mathrm{mJy}$ at $1.4 \mathrm{GHz}$ and $\alpha_{1.4}^{4.85 \mathrm{GHz}}>0.7$. These authors suggested the existence of a large population of short-lived objects that are as yet poorly known. Some of these could be precursors of large-scale FR I galaxies.

To better investigate the nature and properties of compact radio galaxies, we selected from the Bologna Complete Sample (BCS, Giovannini et al. 2001, 2005; Liuzzo et al. 2009b) all sources with a projected linear size smaller than $20 \mathrm{kpc}$. We named this subsample the $\mathrm{C}$ BCS (compact BCS sources) and 
it consists of 18 objects. This complete subsample was selected at low frequency, therefore it should present no bias with respect to the source orientation, possible beaming effects, and spectral index. Most sources in our sample show a moderately steep spectral index, and could not be included in samples such as the one presented by Kunert-Bajraszewska et al. (2010).

The radio power of $\mathrm{C}$ BCS sources is low with respect to powerful FR II or FSRQ, but in the same range as that of giant FR I radio galaxies $\left(10^{23-26} \mathrm{~W} / \mathrm{Hz}\right.$ at $\left.408 \mathrm{MHz}\right)$. Some C BCSs were previously analyzed by us in the radio band (Giroletti et al. $2005 \mathrm{~b}$ ). We present in this paper radio data of the five remaining galaxies with new high-resolution Very Large Array (VLA) observations. We also discuss their optical properties, showing for the first time their optical spectra, since the emission lines analysis is fundamental for understanding the nature and properties of these objects.

Moreover, we include in this paper data of a few extended BCS sources to facilitate a comparison with the C BCS radio and optical properties with different radiogalaxy types (e.g., FRII and FRI extended sources).

The layout of the paper is the following:

- in Sect. 2, we describe radio and optical data for C BCS sample, in particular the new high resolution VLA images and Telescopio Nazionale Galileo (TNG) observations;

- in Sect. 3, we present optical results for all our targets;

- in Sect. 4, we analyze the relation between the optical and radio emissions;

- in Sect. 5, we report notes on single sources;

- in Sect. 6, we discuss our main results with those in the literature;

- in Sect. 7, we summarize our main conclusions.

Throughout this paper, we use $\mathrm{H}_{0}=70 \mathrm{~km} \mathrm{~s}^{-1} \mathrm{Mpc}^{-1}, \Omega_{\mathrm{M}}=0.3$ and $\Omega_{\Lambda}=0.7$. Spectral indices are defined such that $S(v) \propto v^{-\alpha}$.

\section{Radio and optical data}

Table 1 summarizes references for radio and optical data of all $\mathrm{C}$ BCS; $\mathrm{N}$ indicates sources for which new data are presented for the first time in this paper. We also added a few extended BCSs with new data presented here, to increase the statistics in the comparison between compact and extended sources.

\subsection{New radio data}

In this section, we describe the data analysis for the new sub-kpc scale VLA data obtained by us for B2 $0149+35$, B2 $0708+32$ B, B2 $0722+30, \mathrm{~B} 21254+27$, and B2 $1557+26$. We also report the new sub-kpc VLA observations of two peculiar BCS radio sources, B2 0331+39 and B2 1512+30.

The new high-resolution VLA observations were obtained in two observing runs in 2006, March 11 and 2006, April 04. The array was in A configuration, and the observing frequencies were $8.4 \mathrm{GHz}$ and $22 \mathrm{GHz}$. Standard observing schedules for high-frequency observations were prepared, including scans to determine the primary reference pointing, and using a short (3 s) integration time and fast switching mode (180 s on source, $60 \mathrm{~s}$ on calibrator) for $K$-band $(22 \mathrm{GHz})$ scans. Post-correlation processing and imaging were performed with the National Radio Astronomy Observatory (NRAO) Astronomical Image Processing System (AIPS). Parameters of natural UV-weighted images are reported in Table 2. Our new VLA images for resolved sources are shown in Sect. 5. To separate the different
Table 1. Upper part: C BCS sources, and Lower part: extended (E BCS) sources with new data published in this paper.

\begin{tabular}{llcc}
\hline \hline Name & Type & $\begin{array}{c}\text { Radio } \\
\text { data }\end{array}$ & $\begin{array}{c}\text { Optical } \\
\text { data }\end{array}$ \\
\hline B2 0116+31 & C BCS & 3 & $\mathrm{~N}$ \\
B2 0149+35 & C BCS & N & 10 \\
B2 0222+36 & C BCS & 5 & $\mathrm{~N}$ \\
B2 0258+35 & C BCS & 5 & $\mathrm{~N}$ \\
B2 0648+27 & C BCS & 5 & $\mathrm{~N}$ \\
B2 0708+32B & C BCS & N & - \\
B2 0722+30 & C BCS & N & 11 \\
B2 1037+30 & C BCS & 5 & N \\
B2 1101+38 & C BCS & 6 & 12 \\
B2 1217+29 & C BCS & 4 & 13 \\
3C 272.1 & C BCS & 1 & 14 \\
B2 1254+27 & C BCS & N & 15 \\
B2 1257+28 & C BCS & 9 & 15 \\
B2 1322+36B & C BCS & 1 & 15 \\
B2 1346+26 & C BCS & 7 & 16 \\
3C 305 & C BCS & 8 & 15 \\
B2 1557+26 & C BCS & N & 15 \\
B2 1855+37 & C BCS & 5 & N \\
\hline B2 0331+39 & E BCS & N & - \\
B2 0844+31 & E BCS & 2 & $\mathrm{~N}$ \\
B2 1003+35 & E BCS & 2 & $\mathrm{~N}$ \\
B2 1144+35 & E BCS & 1 & 15 \\
B2 1512+30 & E BCS & N & N \\
B2 1626+39 & E BCS & 1 & 14 \\
\hline & & &
\end{tabular}

Notes. N: New high-resolution VLA and/or optical data.

References. 1) Giovannini et al. (2001); 2) Giovannini et al. (2005); 3) Giroletti et al. (2003); 4) Giroletti et al. (2005a); 5) Giroletti et al. (2005b); 6) Giroletti et al. (2006); 7) Liuzzo et al. (2009a); 8) Massaro et al. (2009); 9) Liuzzo et al. (2010); 10) Crawford et al. (1999); 11) Morganti et al. (1992); 12) Capetti et al. (2010); 13) Ho et al. (1997); 14) Buttiglione et al. (2009); 15) SDSS DR7, Abazajian et al. (2009); 16) Anton (1993).

source components and relative fluxes, and dimensions, we used the JMFIT task in AIPS, which is a sophisticated least-squares fit of an image with Gaussian components. Taking also spectral index considerations into account, we identified the core as the unresolved (point-like, see Table 5) component that has the higher flux density in the source. If they are listed, the spectral index maps were made with AIPS using the same UV-range. Typical errors for flux density measurements are $\sim 3 \%$ for $8.4 \mathrm{GHz}$, and $\sim 10 \%$ for $22 \mathrm{GHz}$.

\subsection{Optical data}

We collected optical information available for all C BCS sources, new optical spectra and combined with the emission line measurements from the literature. In Table 1 we list references used for our optical analysis. We indicate literature references for optical information with numbers. With $\mathrm{N}$ we indicate sources for which optical observations were taken by us with the TNG, a $3.58 \mathrm{~m}$ optical/infrared telescope located on the Roque de los Muchachos in La Palma Canary Island (Spain). The spectra for the five C BCS objects are available from the Sloan Digital Sky Survey (SDSS) database, Data Release 7 (Abazajian et al. 2009). We added to the sample unpublished optical observations for some extended BCSs to compare the compact BCS sources with the extended ones. We emphasize 
E. Liuzzo et al.: Compact sources in the Bologna Complete Sample: high-resolution VLA observations and optical data

Table 2. Radio observation parameters for BCS sources: compact (upper) and extended (lower).

\begin{tabular}{lcccccc}
\hline \hline Name & $\begin{array}{c}\text { Beam } \\
\left({ }^{\prime \prime} \times{ }^{\prime \prime}\right)\end{array}$ & $\begin{array}{c}8.4 \mathrm{GHz} \\
\text { Noise } \\
(\text { mJy/beam })\end{array}$ & $\begin{array}{c}\text { Peak } \\
(\mathrm{mJy} / \mathrm{beam})\end{array}$ & $\begin{array}{c}\text { Beam } \\
\left({ }^{\prime \prime} \times{ }^{\prime \prime},{ }^{\circ}\right.\end{array}$ & $\begin{array}{c}22 \mathrm{GHz} \\
\text { Noise } \\
(\mathrm{mJy} / \text { beam })\end{array}$ & $\begin{array}{c}\text { Peak } \\
(\mathrm{mJy} / \text { beam })\end{array}$ \\
\hline B2 0149+35 & $0.35 \times 0.30,-86$ & 0.05 & 5.4 & $0.12 \times 0.10,+85$ & 0.09 & 4.2 \\
B2 0708+32B & $0.36 \times 0.30,+60$ & 0.03 & 12.3 & $0.12 \times 0.11,+46$ & 0.06 & 7.3 \\
B2 0722+30 & $0.34 \times 0.32,-88$ & 0.02 & 14.6 & $0.09 \times 0.08,+71$ & 0.08 & 5.1 \\
B2 1254+27 & $0.31 \times 0.30,-56$ & 0.02 & 1.0 & $0.12 \times 0.12,+0$ & 0.06 & 5.2 \\
B2 1557+26 & $0.32 \times 0.32,+60$ & 0.03 & 13.1 & $0.12 \times 0.11,+34$ & 0.06 & 12.5 \\
\hline B2 0331+39 & $0.34 \times 0.29,-43$ & 0.08 & 146.7 & $0.13 \times 0.11,-52$ & 0.07 & 117.1 \\
B2 1512+30 & $0.20 \times 0.20,+40$ & 0.05 & ND & $0.07 \times 0.08,+23$ & 0.06 & ND \\
\hline
\end{tabular}

Notes. ND indicates sources undetected in our new VLA data.

Table 3. Log of the new TNG observations.

\begin{tabular}{lccc}
\hline \hline Name & Redshift & Date & $T_{\text {exp }}$ \\
\hline B2 0116+31 & 0.059 & $2003 / 08 / 22$ & $1800 \times 2$ \\
B2 $0222+36$ & 0.033 & $2003 / 08 / 22$ & 2400 \\
B2 $0258+35$ A & 0.017 & $2003 / 08 / 22$ & 2400 \\
B2 $0648+27$ & 0.041 & $2003 / 09 / 22$ & 2400 \\
B2 $1037+30$ & 0.091 & $2003 / 12 / 17$ & $1800 \times 2$ \\
B2 $1855+37$ & 0.056 & $2003 / 08 / 21$ & $1800 \times 2$ \\
\hline B2 0844+31B & 0.067 & $2003 / 11 / 14$ & 2400 \\
B2 1003+35 & 0.099 & $2003 / 11 / 18$ & $1800 \times 2$ \\
B2 1512+30 & 0.094 & $2003 / 08 / 23$ & $1800 \times 2$ \\
\hline
\end{tabular}

Notes. Column 1: target names. Upper sources are C BCS while sources below the solid line are extended BCSs; Col. 2: redshift of the sources; Col. 3: date of observation; Col. 4: the exposure time in sec where $\times 2$ means that the image was repeated.

considered all optical information available up to now for the whole BCS sample.

\subsubsection{TNG observations}

Table 3 contains the journal of observations and basic information for our targets. The TNG observations were made using the Device Optimized for the LOw RESolution (DOLORES) or the Low Resolution Spectrograph (LRS) installed at the Nasmyth B focus of the telescope. The detector is a Loral-thinned and backilluminated $2048 \times 2048$ CCD. Observations were performed in service mode during several nights between August 21th and December 18th 2003. The seeing, measured from the acquisition images, varied between 0.8 arcsec and 1.3 arcsec. We used the low-resolution LR-B grism and the 1.0 arcsec slit width. The dispersion is $2.8 \AA$ per pixel and the spectral resolution about $11 \AA$. The typical useful spectral range is $\sim 3500$ to $\sim 8000 \AA$ with increasing fringing beyond $7200 \AA$. This range of wavelengths enables us to analyze all relevant emission lines of the optical spectrum: $\mathrm{H} \beta,[\mathrm{OIII}] \lambda \lambda$ 4959, $5007 \AA$, [OI] $\lambda \lambda$ 6300, $64 \AA, \mathrm{H} \alpha$, [NII] $\lambda \lambda$ 6548, $84 \AA$, and [SII] $\lambda \lambda$ 6716, $31 \AA$.

\subsubsection{Data analysis}

The data were reduced using the LONGSLIT package of with the National Optical Astronomy Observatory (NOAO) reduction software. IRAF (Image Reduction and Analysis Facility) is distributed by the National Optical Astronomy Observatories, which are operated by the Association of Universities for Research in Astronomy, Inc., under cooperative agreement with the National Science Foundation. It is available at http:// iraf. noao. edu/. A bias frame was subtracted from any frame, then the flat field correction was applied to remove the pixel-topixel gain variations. After that, the wavelength calibration, the optical distortion corrections, and the background-subtraction were applied. One-dimensional spectra were extracted by summing in the spatial direction over an aperture corresponding to the nuclear part of the source: we extracted and summed the six pixel rows closest to the center of the spectrum, corresponding to $1.65^{\prime \prime}$. Lastly the relative flux calibration was made using spectro-photometric standard stars observed during each night. In Fig. 1 we present the C BCS source optical spectra after the calibration.

To properly measure the emission lines intensities, we needed to subtract the stellar emission of the host galaxies. Before removing the stellar continuum, we corrected for reddening from the Galaxy (Burstein et al. 1982, 1984) using the extinction law of Cardelli et al. (1989). The galactic extinction $E(B-V)$ used for each object was taken from the NASA Extragalactic Database (NED) database. The adopted method consists on modeling the nuclear spectra with a single stellar population taken from the Bruzual et al. (2003) library and then subtracting the best fit model spectrum from the nuclear one. The templates assume a Salpeter initial mass function (IMF) formed in an instantaneous burst, with solar-metallicity stars in the mass range $0.1 \leq M \leq 125 M_{\odot}$. The parameters were left free to vary independently of each other to obtain the best fit of the stellar age (from 1 to $13 \mathrm{Gyr}$ ), the metallicity (from 0.0008 to 0.5 solar metallicity), the normalization of the model, the velocity dispersion, the continuum emission from the active galactic nucleus (AGN), and its slope. Although this method of stellar removal gives also an estimate of the velocity dispersion, for the resolution of our spectra this value is dominated by the instrumental broadening. The spectral regions chosen for the fit are centered on the $\mathrm{H} \beta$ and $\mathrm{H} \alpha$ emission lines, with a range of $3600-5500 \AA$ for the $\mathrm{H} \beta$ and 5700-7100 $\AA$ for the $\mathrm{H} \alpha$. The emission lines were excluded from the fit, since they are strongly affected by the nuclear emission, even more so than the stellar emission. Other small regions were excluded because of telluric absorption, cosmic rays, or other impurities. At the end of this operation, we obtained the nuclear emission produced by the AGN activity only. In Fig. 2 we show as an example of the adopted procedure the spectra of two C BCS sources. The source spectra are depicted as solid lines, the top spectra are before the 

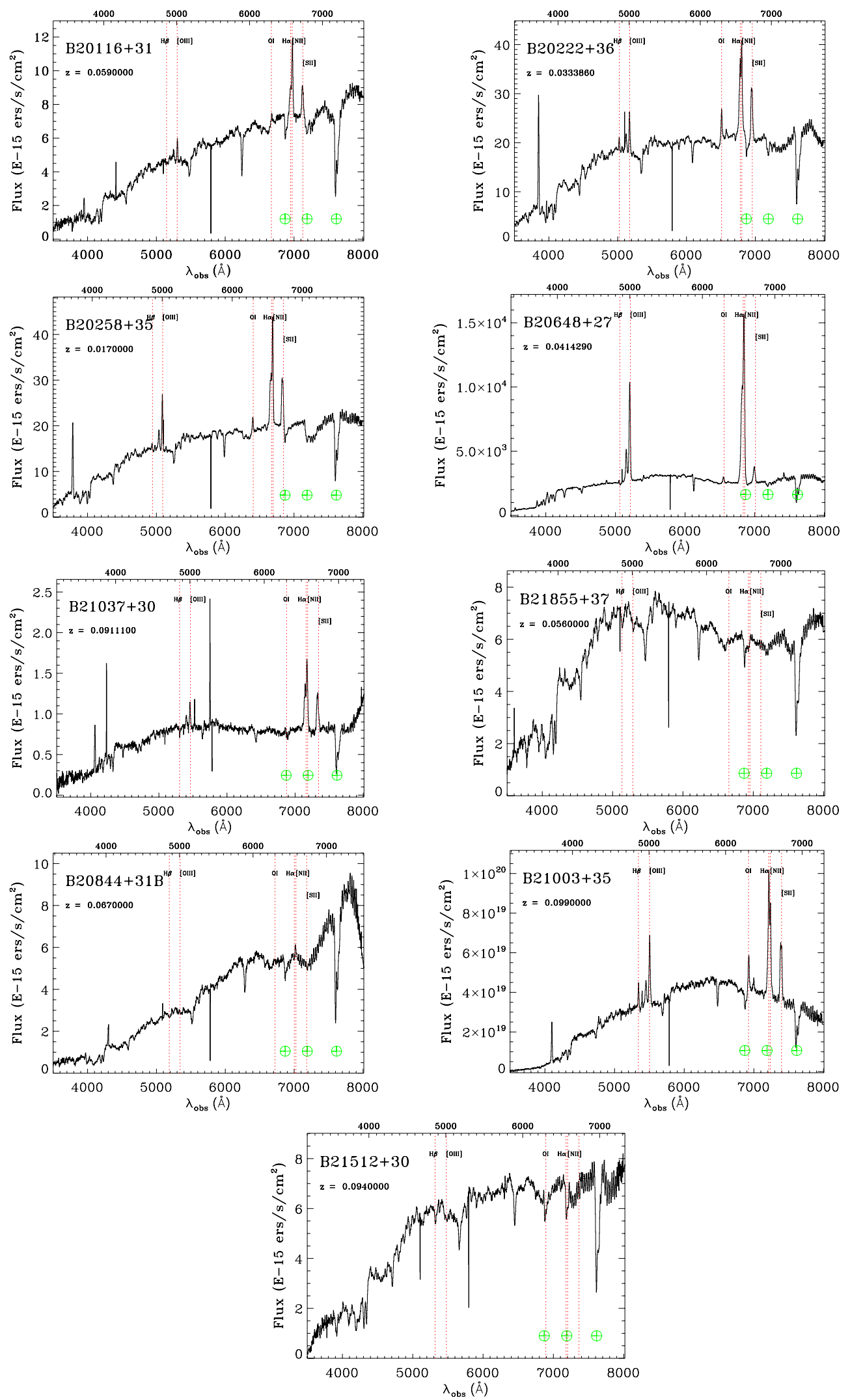

Fig. 1. Optical spectra of our TNG targets. These spectra are extracted from the central 1.65 arcsec of the galaxies. The fluxes are in units of $10^{-15} \mathrm{erg} \mathrm{s}^{-1} \mathrm{~cm}^{-2}$. The red dotted vertical lines indicate the position of the main optical emission lines, while the green crossed circles on the bottom of the spectra indicate the position of the main telluric absorption lines. On the upper left side of the spectra the source redshift is indicated below the target name.

stellar removal and the bottom spectra are the results of the host galaxy stellar population subtraction. The dotted line through the top spectra indicates the single stellar population model. The dashed line across the bottom spectra indicates the zero flux level. These spectra have quite a flat continuum emission with the overlap of emission lines produced by the photoionised gas.

\section{Optical results}

\subsection{Measure of the emission line luminosities}

In Table 4 we report the emission lines fluxes. Line intensities of targets observed with the TNG are measured using the 
Table 4. New TNG emission line fluxes.

\begin{tabular}{|c|c|c|c|c|c|c|c|}
\hline Name & $\mathrm{H} \alpha$ & {$[\mathrm{NII}] \lambda 6584 \AA$} & [OI] $\lambda 6364 \AA$ & {$[\mathrm{SII}] \lambda 6716 \AA$} & {$[\mathrm{SII}] \lambda 6731 \AA$} & $\mathrm{H} \beta$ & {$[\mathrm{OIII}] \lambda 5007 \AA$} \\
\hline $\bar{B} 20116+31$ & $(3.9 \pm 0.4)$ & $(9.2 \pm 0.4)$ & $(2.5 \pm 0.3)$ & $(5.4 \pm 0.4)$ & $(2.5)$ & $(0.54 \pm 0.09)$ & $(3.05 \pm 0.11)$ \\
\hline B2 $0222+36$ & $(3.3 \pm 0.1) \mathrm{e}+1$ & $(4.0 \pm 0.1) \mathrm{e}+1$ & $(1.7 \pm 0.1) \mathrm{e}+1$ & $(2.1 \pm 0.1) \mathrm{e}+1$ & $(1.7) \mathrm{e}+1$ & $(9.2 \pm 0.4)$ & $(2.10 \pm 0.05) \mathrm{e}+1$ \\
\hline B2 $0258+35 \mathrm{~A}$ & $(3.1 \pm 0.1) \mathrm{e}+1$ & $(6.4 \pm 0.1) \mathrm{e}+1$ & $(1.8 \pm 0.1) \mathrm{e}+1$ & $(3.2 \pm 0.1) \mathrm{e}+1$ & $(1.7) \mathrm{e}+1$ & $(8.1 \pm 0.9)$ & $(3.8 \pm 0.1) \mathrm{e}+1$ \\
\hline B2 $0648+27$ & $(1.4 \pm 0.1) \mathrm{e}+4$ & $(3.55 \pm 0.02) \mathrm{e}+4$ & $(1.9 \pm 0.2) \mathrm{e}+3$ & $(2.3 \pm 0.2) \mathrm{e}+3$ & (1.9)e +3 & $(8.7 \pm 0.8) \mathrm{e}+3$ & $(9.88 \pm 0.01) \mathrm{e}+4$ \\
\hline B2 $1037+30$ & $(1.00 \pm 0.05)$ & $(1.7 \pm 0.05)$ & $(3.2 \pm 0.4) \mathrm{e}-1$ & $(8.4 \pm 0.6) \mathrm{e}-1$ & $(3.2) \mathrm{e}-1$ & $(0.4 \pm 0.2) \mathrm{e}-1$ & $(6.0 \pm 0.3) \mathrm{e}-1$ \\
\hline B2 $1855+37$ & - & $<7.8 \mathrm{e}-1$ & - & - & - & - & - \\
\hline B2 0844+31B & $(7.5 \pm 1.5) \mathrm{e}-1$ & $(1.5 \pm 0.2)$ & - & - & - & - & $(2.1 \pm 0.3)$ \\
\hline B2 $1003+35$ & $(6.5 \pm 0.1)$ & $(4.66 \pm 0.08)$ & $(2.3 \pm 0.1)$ & $(3.51 \pm 0.09)$ & $(2.33)$ & $(1.5 \pm 0.1)$ & $(4.8 \pm 0.1)$ \\
\hline B2 $1512+30$ & - & - & - & - & - & $<2.0 \mathrm{e}-1$ & $(5.1 \pm 0.9) \mathrm{e}-1$ \\
\hline
\end{tabular}

Notes. The sources are listed in the first column; in the following columns the fluxes of the major emission lines are listed. The fluxes are given in units of $10^{-15} \mathrm{erg} \mathrm{s}^{-1} \mathrm{~cm}^{-2}$. The upper limits to the line measurements are indicated by $<$.
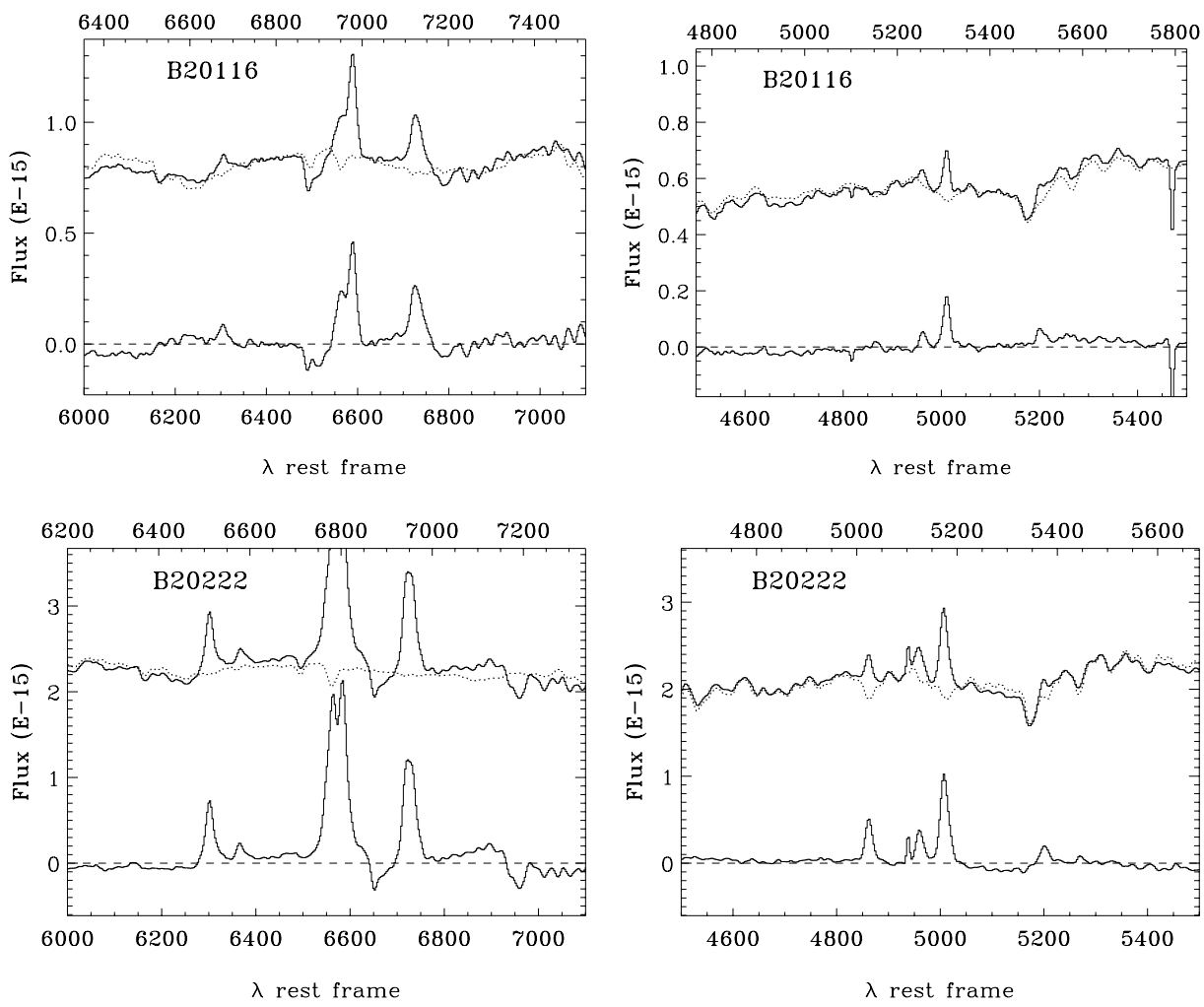

Fig. 2. Spectra of two C BCSs after the subtraction of the single stellar population model with the best fit. The first column represents the modeling centered on the $\mathrm{H} \alpha$ line, while the second column shows the fits for the $\mathrm{H} \beta$ line. In all panels, the $\mathrm{C}$ BCS spectra are depicted in solid lines, the top spectra show the status before the stellar removal and the bottom spectra are the results of the host galaxy stellar population subtraction. The dotted line through the top spectra indicates the bestfit single stellar population model. The dashed line across the bottom spectra indicates the zero flux level.

specfit package to fit Gaussian profiles to each emission line in our sources: $\mathrm{H} \beta$, [OIII] $\lambda \lambda 4959.5007 \AA$, [OI] $\lambda \lambda$ $6300.64 \AA, \mathrm{H} \alpha$, [NII] $\lambda \lambda 6548.84 \AA$, and [SII] $\lambda \lambda 6716.31 \AA$. To reduce the number of free parameters, we forced the velocity and full width at half maximum (FWHM), to be the same for all the lines. The integrated fluxes of each line were left free to vary except for those with known ratios from atomic physics, which were the $[\mathrm{OI}] \lambda \lambda$ $6300.64 \AA$, [OIII] $\lambda \lambda 4959.5007 \AA$ and [NII] $\lambda \lambda 6548.84 \AA$ doublets. Where required, we inserted a flat continuum. In some cases, we tried to fit a broad component for both the $\mathrm{H} \beta$ and $\mathrm{H} \alpha$ lines, but the resulting lines intensities were too low with respect to the narrow components and were excluded. For objects with information available in literature, we adopted the emission line measurements as reported by the authors (see references in Table 1).

\subsection{Diagnostic diagrams}

Diagnostic diagrams are constructed from pairs of observed line ratios, which reveal information on ionizing continuum, ionization parameter, gas temperature, and other physical properties of the emission line regions. According to Heckman (1980), Baldwin et al. (1981), Kewley et al. (2006), and other works, star-forming galaxies and AGNs are separated, with the latter additionally separated into high-excitation galaxies (HEG) and low-excitation galaxies (LEG). These ratios are chosen so that considered lines are very close to avoid reddening and extinction problems (strongest for the bluer part of the spectrum), and the ratios can also be measured even if there are uncertainties on the flux calibration of the spectra. Moreover, the chosen lines are often the strongest features of the optical spectra, easily found also in low-luminosity galaxies.

In Fig. 3 we show the three diagnostic diagrams in Table 1: $([\mathrm{OIII}] \lambda 5007 / \mathrm{H} \beta)$ versus $([\mathrm{NII}] \lambda 6583 / \mathrm{H} \alpha),([\mathrm{OIII}] \lambda 5007 / \mathrm{H} \beta)$ versus ([SII] $\lambda 6716 \lambda 6731 / \mathrm{H} \alpha)$ and the $([\mathrm{OIII}] \lambda 5007 / \mathrm{H} \beta)$ versus $([\mathrm{OI}] \lambda 6364 / \mathrm{H} \alpha)$. The standard optical diagnostic diagram is the $([\mathrm{OIII}] \lambda 5007 / \mathrm{H} \beta)$ versus ([NII] $\lambda 6583 / \mathrm{H} \alpha)$. These line ratios are used to separate the star-forming galaxies from AGNs, since the $[\mathrm{NII}] / \mathrm{H} \alpha$ is more sensitive to the presence of lowlevel AGN than other lines due to its sensitivity to metallicities, while the $[\mathrm{OIII}] / \mathrm{H} \beta$ line ratio is sensitive to the ionization 


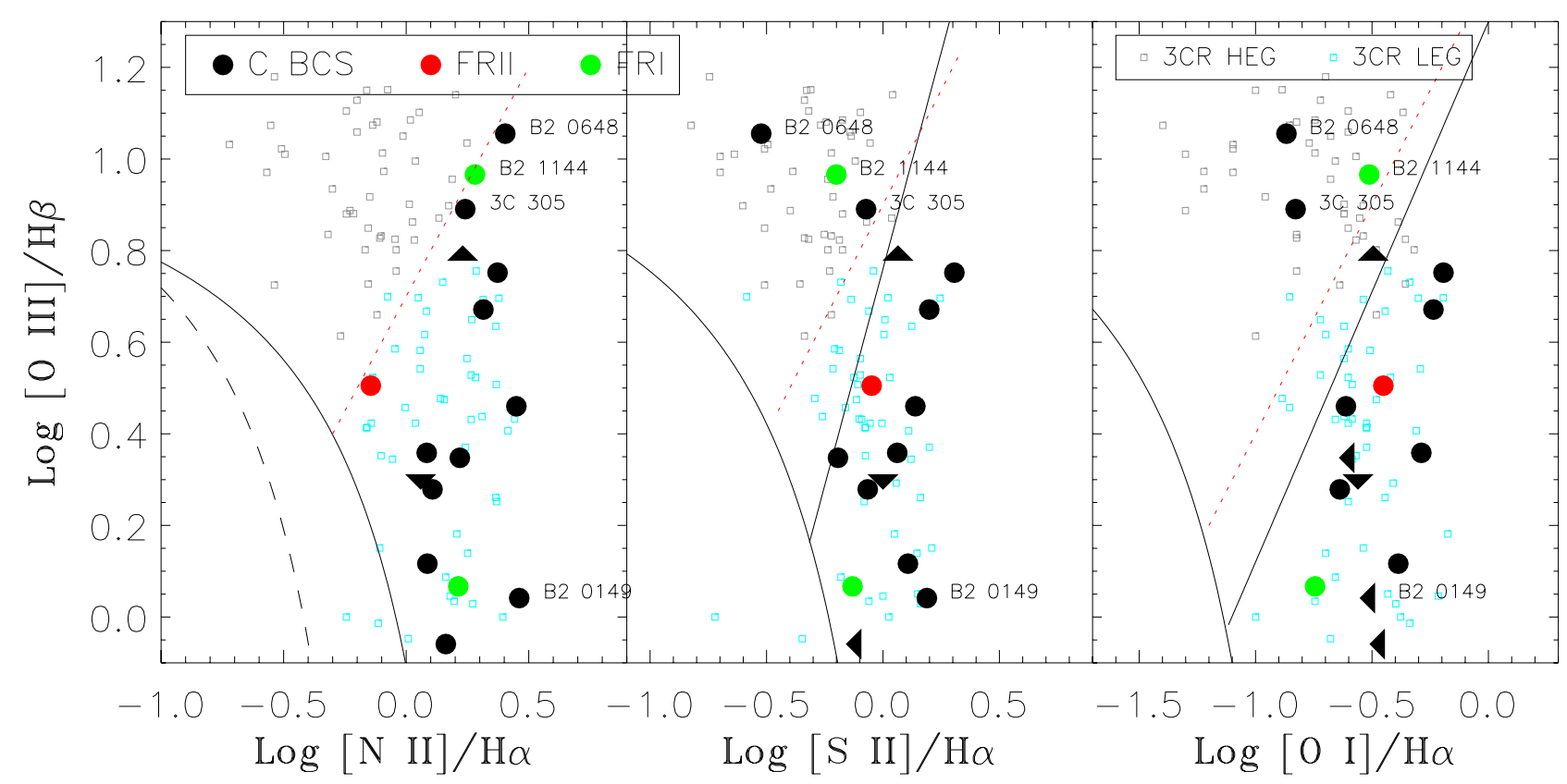

Fig. 3. Diagnostic diagrams: the curves are taken from Kewley et al. (2006) and divide galaxies into star-forming, HEG and LEG AGNs. The region between the dashed line and the solid line in the first diagram indicates the position of composite galaxies. The dotted lines mark the empirical boundaries between HEG (gray squares) and LEG (cyan squares) in each diagram as derived for the 3CR sample (Buttiglione et al. 2010). The C BCS are indicated with black circles, FR II E BCS with red circles and FR I E BCS with green circles (see Table 5). Upper limits (UL) on the emission line measurements are indicated by triangles pointing toward them (i.e. an UL in $\mathrm{H} \beta$ is indicated with a triangle pointing up).

parameter of the gas (the amount of ionization transported by the radiation moving through the gas). The $([\mathrm{OIII}] \lambda 5007 / \mathrm{H} \beta)$ versus $([\mathrm{SII}] \lambda 6716 \lambda 6731 / \mathrm{H} \alpha)$ and the $([\mathrm{OIII}] \lambda 5007 / \mathrm{H} \beta)$ versus $([\mathrm{OI}] \lambda 6364 / \mathrm{H} \alpha)$ diagnostic diagrams are more sensitive to the hardness of the ionizating radiation field, which divides the AGNs into two branches: high-ionization sources (i.e. $H E G$ ) lie on the upper branch, low-ionization sources (i.e. $L E G$ ) lie on the lower branch.

In Fig. 3, B2 0708+32B, B2 0722+30, B2 1257+28, B2 $1855+37$, B2 $0331+39$, B2 0844+31, B2 1101+38, and B2 $1512+30$ were not considered due to their upper limits or undetected lines in at least one of the diagnostic ratios. The starforming HEG and LEG regions are separated by solid lines according to Kewley et al. (2006). The region between the dashed line and the solid line in the $\log ([\mathrm{OIII}] \lambda 5007 / \mathrm{H} \beta)$ versus $\log ([\mathrm{NII}] \lambda 6583 / \mathrm{H} \alpha)$ diagram indicates the region of composite galaxies, sources with both star-forming and nuclear activity. We also plotted in color the HEG (gray) and LEG (cyan) regions occupied by the radio-loud AGN with redshift $z<0.3$ taken from the 3CR (Third Cambridge Catalog of Radio Sources) sample (Buttiglione et al. 2010). The red dotted lines represent the HEG/LEG separation derived for the 3CR. The 3CR is characterized by unbiased selection criteria with respect to optical properties and orientation, and it spans a relatively wide range in redshift and radio power, covering the whole range of behavior of radio-loud AGN. The BCS sources, with sufficiently strong emission lines to make diagnostic diagrams (see Table 4), are shown as colored dots according to their classification. The position of $\mathrm{C}$ BCS sources on the diagnostic diagrams indicates that the majority of our sources belongs to the LEG group. The only exceptions are B2 0648+27 and 3C 305, located in the HEG region. We note also that the BCS FRI radiogalaxy B2 1144+35 is present in this region. The radiogalaxy B2 $0149+35$ is a source in a low-activity phase which is in detail discussed in Sect. 5.1.2.

\section{Optical-radio correlation}

The correlation between the AGN optical narrow emission line and radio power has been verified for a long time (e.g., Baum et al. 1989a,b). This correlation is explained thinking of a common energy source for both the optical lines and radio emission: the isotropically emitted radiation from the active nucleus ionizes the optical emitting gas and the radio luminosity is determined by the properties of the central engine.

As shown by Morganti et al. (1997), the same optical-radio relation holds both for extended sources and compact sources. These authors compared the [OII] $\lambda 3727 \AA$ and [OIII] $\lambda 5007 \AA$ emission line luminosities of a sample of CSS sources with the values found for extended sources of similar radio power and redshift. They found a very intriguing result: in the correlation between the [OII] $\lambda 3727 \AA$ - radio luminosities, both compact and extended radio sources lie on the same linear correlation, while, looking at the [OIII] $\lambda 5007 \AA$-radio luminosities, compact sources tend to be at the lower end of the [OIII] $\lambda 5007 \AA$ luminosity.

In Figs. 4 and 5 we similarly compared the $[\mathrm{OIII}] \lambda$ $5007 \AA-408 \mathrm{MHz}$ radio extended luminosities and [OIII] $\lambda$ $5007 \AA-5 \mathrm{GHz}$ radio core luminosities plots. We included $\mathrm{C}$ and E BCS sources with available optical data, comparing our results with different samples. B2 $0708+32 \mathrm{~B}$ and B2 $0331+39$ are not included because we have no information on the [O III] emission line flux. Because we used a combination of our TNG, SDSS, and other telescope spectra, a potential issue concerning our optical spectroscopic data is the difference in the spatial size of the associated spectral aperture. This is due to the different angular sizes of the aperture and also to the range of redshift covered by our sample. However, we verified that no link is present between the instrumental setup (or redshift) and the location of the various objects in Figs. 4 and 5. 
E. Liuzzo et al.: Compact sources in the Bologna Complete Sample: high-resolution VLA observations and optical data
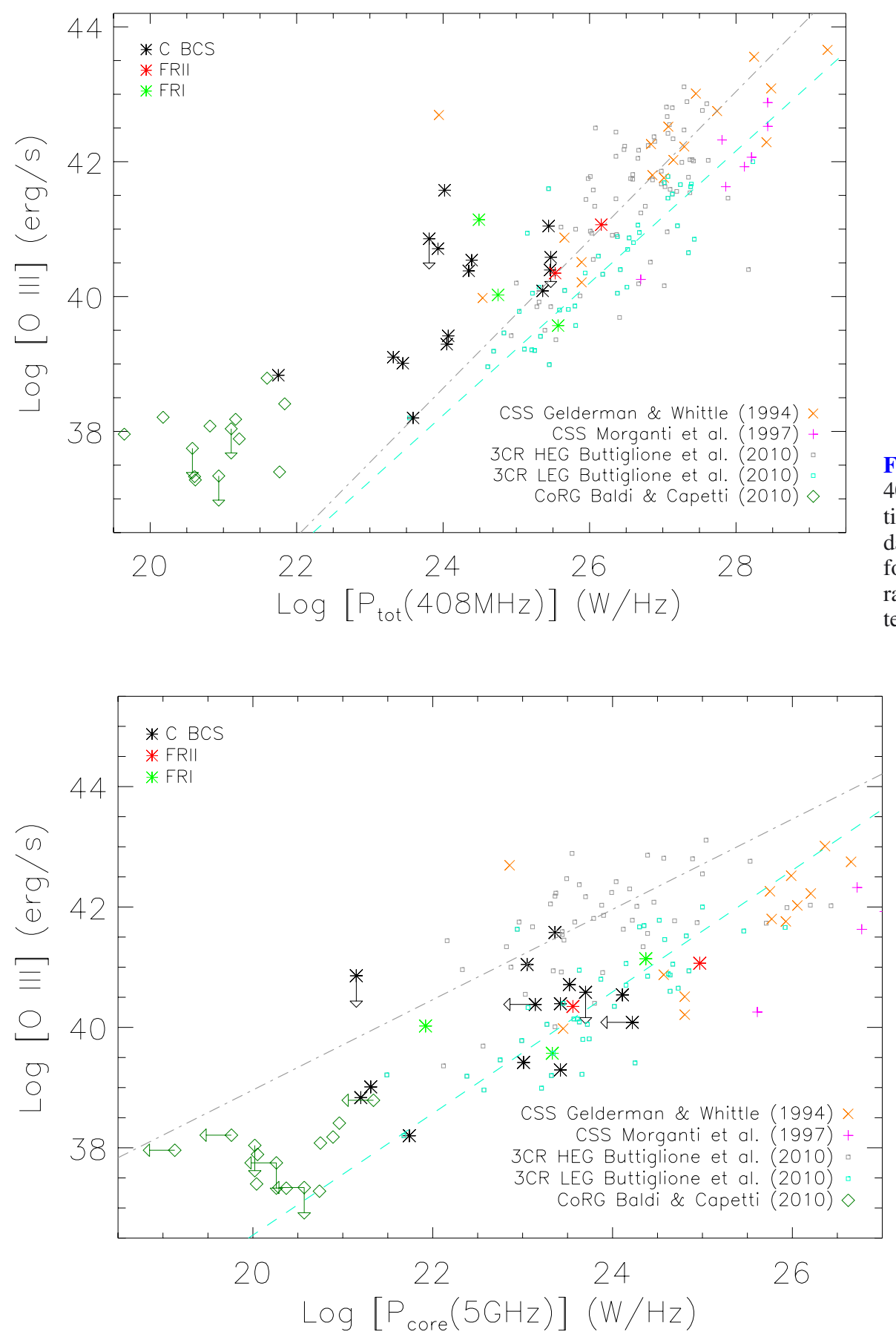

Fig. 4. Correlation of the total radio power at $408 \mathrm{MHz}$ vs. the [O III] emission line intensities produced by the narrow line regions. The two dashed lines represent the best linear fit obtained for the HEG and the LEG sub-populations separately (according to Buttiglione et al. 2010). See text for more details.
Fig. 5. [O III] luminosity $\left[\mathrm{erg} \mathrm{s}^{-1}\right]$ as a function of radio core power at $5 \mathrm{GHz}$ in $\mathrm{W} \mathrm{Hz}^{-1}$ : the two dashed lines represent the best linear fit obtained for the HEG and LEG subpopulations separately according to Buttiglione et al. (2010). Symbols and colors show different samples as in Fig. 4.
We added samples of more powerful compact sources (orange crosses: Gelderman et al. 1994; magenta plus: Morganti et al. 1997), to verify the optical-radio correlation. Moreover, we superimposed the 3CR LEG and HEG sources (gray and cyan small squares, Buttiglione et al. 2010) to compare compact sources with extended ones. Finally, we also plot (green diamonds) the core radio galaxies (CoRG) sample of Baldi \& Capetti (2010) to compare our sample with these peculiar faint compact sources.

From Fig. 4, we note that C BCS sources show a lower total radio power than the $\mathrm{LEG}$ objects discussed by Buttiglione et al. (2010) or that their optical emission line is higher than expected from the radio power. The C BCS sources show a significantly higher optical luminosity with respect to the best linear fit obtained by Buttiglione et al. (2010) for HEG and LEG sources (see cyan and green dashed lines). The CoRGs studied by Baldi \& Capetti (2010) follow the same trend as the C BCS. It looks like that core radio galaxies and $\mathrm{C}$ BCS have in general a lower total radio power than HEG, LEG, and CSS radio sources, but the line optical luminosity is a factor two higher with respect to the correlation with the total radio power.

We also compared at the [OIII] $\lambda 5007 \AA$ luminosity with the $5 \mathrm{GHz}$ radio core luminosities for all samples (Fig. 5). In this diagram CoRGs as well as C BCS sources are in between the HEG and LEG best linear fit. Our C BCS sources seem to be intermediate objects between 3CR LEG + CSS sources and the CoRGs of Baldi \& Capetti (2010). The nuclear emission of the 3CR HEG sources is definitely brighter in [OIII] luminosity, 
Table 5. Sources parameters from the new VLA observations.

\begin{tabular}{|c|c|c|c|c|c|c|c|}
\hline Source & Component & $\begin{array}{c}\text { RA (J2000) } \\
\text { h m s }\end{array}$ & $\begin{array}{c}\operatorname{Dec}(\mathrm{J} 2000) \\
\mathrm{d}^{\prime}{ }^{\prime \prime \prime}\end{array}$ & $\begin{array}{c}8.4 \mathrm{GHz} \\
\text { Flux } \\
(\mathrm{mJy})\end{array}$ & $\begin{array}{c}22 \mathrm{GHz} \\
\text { Flux } \\
(\mathrm{mJy})\end{array}$ & $\alpha_{8.4}^{22}$ & $\begin{array}{l}\text { Size } \\
\prime \prime\end{array}$ \\
\hline B2 0149+35 & core & 015246.458 & 360906.50 & 5.5 & 4.9 & 0.12 & $\mathrm{P}$ \\
\hline \multirow[t]{4}{*}{ B2 $0708+32 B$} & core & 071147.704 & 321835.12 & 12.6 & 7.5 & 0.54 & $\mathrm{P}$ \\
\hline & $\mathrm{N}$ lobe & - & - & 2.7 & - & - & 3.5 \\
\hline & S lobe & - & - & 3.2 & - & - & 3.0 \\
\hline & total & - & - & 18.5 & - & - & 13.0 \\
\hline \multirow[t]{3}{*}{ B2 $0722+30$} & core & 072537.256 & 295714.96 & 14.6 & 6.7 & 0.77 & $\mathrm{P}$ \\
\hline & E lobe & - & - & 4.9 & - & - & 8.0 \\
\hline & total & - & - & 19.0 & 6.7 & 1.08 & 12.0 \\
\hline \multirow[t]{2}{*}{ B2 1254+27 } & core & 125724.356 & 272952.23 & 1.1 & 5.3 & -1.63 & $\mathrm{P}$ \\
\hline & total & - & - & 1.2 & 5.6 & -1.60 & 0.3 \\
\hline \multirow[t]{3}{*}{ B2 $1557+26$} & core & 155951.616 & 255626.35 & 13.5 & 12.3 & 0.10 & $\mathrm{P}$ \\
\hline & NE jet & - & - & 1.8 & - & - & 0.7 \\
\hline & total & - & - & 15.3 & 12.3 & - & 0.6 \\
\hline \multirow[t]{4}{*}{ B2 $0331+39$} & core & 033418.415 & 392124.37 & 145.8 & 114.5 & 0.25 & $\mathrm{P}$ \\
\hline & $S$ jet & - & - & 28.9 & 13.9 & 0.76 & 0.9 \\
\hline & halo & - & - & 29.5 & - & - & 3.5 \\
\hline & total & - & - & 204.2 & 136.2 & - & 3.5 \\
\hline
\end{tabular}

Notes. In Col. 8, P indicates point-like structure and it is related to the core morphology (see text in Sect. 2.1).

while $\mathrm{C} B C S$ at a given core radio power are near to the linear best fit of LEG sources, but on average C BCS are optically brighter.

Furthermore CSS sources do not follow the two linear fits, which suggests a possible common fit of CSS, C BCS, and core radio galaxies. Evidently, they follow an independent track with respect to HEG and LEG sources. Kunert-Bajraszewska et al. (2010), discussing a sample of low-luminosity compact objects, suggest two parallel evolutionary tracks for HEG and LEG sources, evolving from GPS (gigahertz peaked sources) to CSS to FR. Our diagram, thanks to the addition of low-power core radio galaxies and $\mathrm{C} B \mathrm{BCS}$ sources selected at low frequency and without any constrain on their spectral index, suggests a more complex scenario.

\section{Notes on single sources}

\subsection{Compact BCS sources}

In this section, we give short notes on the whole sample of C BCSs. For all resolved targets in our new radio maps, we also present an image. For all these sources, if not specified otherwise, the beam size and the noise level of each map are those reported in Table 2. In Table 5, we give basic source parameters at $8.4 \mathrm{GHz}$ and $22 \mathrm{GHz}$ for our new VLA images. The reported core positions are obtained from our new VLA observations.

The arcsecond core flux density at $5 \mathrm{GHz}$ and total flux density at $408 \mathrm{MHz}$ are from Liuzzo et al. (2009b).

\subsubsection{B2 $0116+31$}

The radio galaxy B2 $0116+31(4 \mathrm{C} 31.04)$ is classified as a low-redshift CSO (compact symmetric object) (Giovannini et al. 2001; Cotton et al. 1995). VLBA (Very Long Baseline Array) images at $5 \mathrm{GHz}$ show a compact core component with hot spots and lobes on either side (Giroletti et al. 2003). Spectral line VLBI (Very Long Baseline Interferometer) observations reveal the presence of an edge-on circumnstellar H I disk (Perlman et al. 2001). The optical nucleus is also found to have cone-like features well aligned with the radio axis.
According to Perlman et al. (2001), optical data suggest that a relatively recent merger has occurred $\gtrsim 10^{8} \mathrm{yr}$ ago. In our TNG spectrum we detect all diagnostic emission lines and their ratios clearly settle the source among LEG.

\subsubsection{B2 $0149+35$}

B2 $0149+35$ is identified with NGC 708 , a D/cD galaxy associated with the brightest galaxy in the central cooling flow cluster Abell 262 (Braine \& Dupraz 1994). B2 0149+35 is a low-brightness galaxy whose nuclear regions are crossed by an irregular dust lane and dust patches (Capetti et al. 2000).

The interaction between the cooling gas and the radio source is discussed by Blanton et al. (2004). The comparison between the total and core radio power and between radio and X-ray images suggests that at present the radio core is in a low-activity phase (Blanton et al. 2004; Liuzzo et al. 2010).

Our VLA observations show an unresolved component with a flux density of $5.5 \mathrm{mJy}$ at $8.4 \mathrm{GHz}, 4.9 \mathrm{mJy}$ at $22 \mathrm{GHz}$, and a flat spectrum $\left(\alpha_{22}^{8.4} \sim 0.12\right)$. The lower flux density in $5 \mathrm{GHz}$ VLBA maps can be due to a slightly inverted spectrum or to some extension lost in VLBA images and/or because of variability.

From the diagnostic diagrams and the [O III]-radio plots the source is classified as LEG.

\subsubsection{B2 $0222+36$}

This source shows an halo-core structure at arcsecond resolution (Fanti et al. 1986). Sub-arcsecond $8 \mathrm{GHz}$ VLA maps resolve the structure into a core and two components on either side, while at $22 \mathrm{GHz}$ it shows an S-shaped morphology with a dominant core and two lobes (Giroletti et al. 2005b).

In VLBI images at $1.6 \mathrm{GHz}, \mathrm{B} 20222+36$ is two sided, with jets emerging in opposite directions along the north-south axis. Since there is evidence of a change of the jet direction in the inner region, Giroletti et al. (2005b) speculated that the jet orientation is rotating because of instabilities of the AGN. This could explain the small size of the source because an unstable jet 
would not allow the growth of a large scale radio galaxy. In this case the old round halo could be caused by the diffusion of radio emission during the orbit of the inner structure.

From the diagnostic diagrams, this source is classified as an LEG-type.

\subsubsection{B2 0258+35}

This source was studied with VLA and Merlin + European VLBI Network (EVN) by Sanghera et al. (1995), who classified it as a CSS source. VLA data show a double structure with a separation of 1.1". The EVN + MERLIN images reveal an extended plume-like feature at both ends of the source and a jet-like feature in between. Sub-arsecond VLA images at 8 and $22 \mathrm{GHz}$ reveal the same structure. The source appears to strongly interact with the ISM (interstellar medium) as shown by the large bending of the arcsecond structure of the SE lobe and the presence of a surrounding low-brightness extended structure in the VLA images. A large amount of an extended HI-disk in the central region of the source is detected (Emonts et al. 2006a,b).

From the diagnostic diagrams, it is an LEG-type source.

\subsubsection{B2 0648+27}

This object is only slightly extended at the lowest frequencies (Parma et al. 1986). It was resolved into a double source extended about 1 " with VLA observations at $8.4 \mathrm{GHz}$ (Giroletti et al. 2005b). Morganti et al. (2003) detected a large amount of $\mathrm{H}$ I. The two radio lobes do not show any evidence of a jet-like structure or hot spots.

Giroletti et al. (2005b) estimated a minimum age for this source of about 1 Myr. The external lobe regions are probably much older, confirming that this source is confined, and it is expected to remain compact similar to NGC 4278 (Giroletti et al. 2005a). The connection between the small size of the radio emission and major merger in this galaxy about $10^{9}$ years ago, with the large amount of H I in this galaxy (Morganti et al. 2003) is remarkable.

From the optical spectrum, we observe very strong highexcitation emission lines and the Balmer lines in absorption, as the spectra produced by a dominant young stars population. In the diagnostic diagrams, this source occupies the region of HEG galaxies. This is the most powerful source in optical band in our sample.

\subsubsection{B2 0708+32B}

This source reveals an extended morphology in NS direction (Fanti et al. 1986) with two symmetric lobes about $10^{\prime \prime}$ in size ( $\sim 10 \mathrm{kpc})$. In VLBA images at $5 \mathrm{GHz}$ (Liuzzo et al. 2009b), a double structure oriented along PA (position angle) $\sim 150^{\circ}$ and extended $\sim 4$ mas is observed. Since no spectral index information is available, the identification of the core is not possible.

Our 8.4 GHz map (Fig. 6) shows an unresolved nucleus (Table 5) and two symmetric lobes in agreement with the structure present in the $1.4 \mathrm{GHz}$ observations. No jet-like structure is visible. In our $22 \mathrm{GHz}$ VLA observation, only the core is visible (Table 5). The angular resolution of the VLA images is too low to resolve the double structure that is visible in VLBA images. Using VLA data at $1.4 \mathrm{GHz}$ from Fanti et al. (1986), we estimated the spectral index for lobes and central component. We derived $\alpha_{\text {lobes }} \sim 1$ for the two lobes and for the central component: $\alpha_{1.4}^{8.4}=0.3$ and $\alpha_{8.4}^{22}=0.54$. The steepening

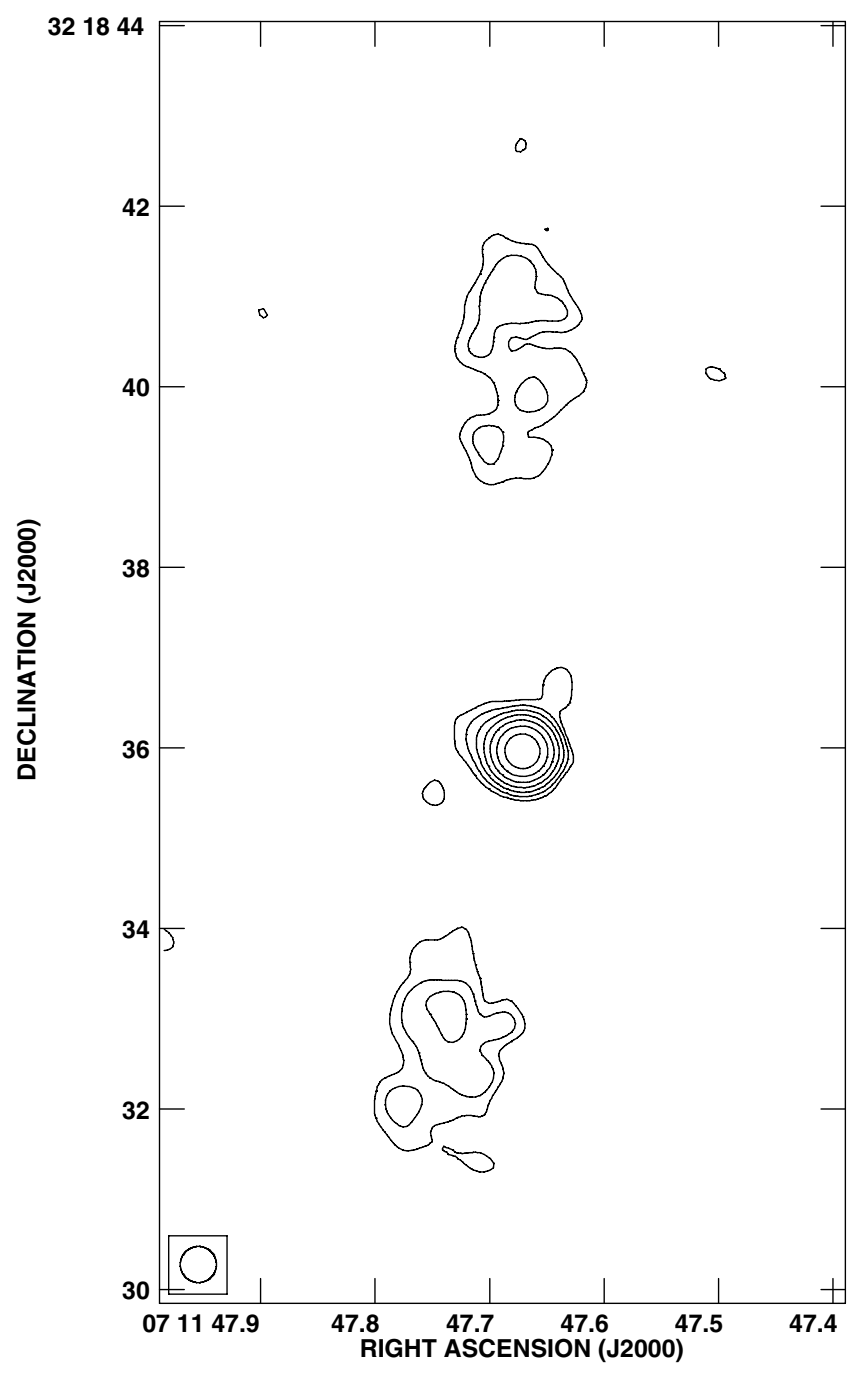

Fig. 6. 8.4 GHz VLA image of B2 $0708+32 \mathrm{~B}$. Contour levels are 0.1, $0.2,0.4,0.8,1.6,3.2,6.4$, and $12.8 \mathrm{mJy} / \mathrm{beam}$. The peak level is $11.8 \mathrm{mJy} /$ beam. The beam size is $0.4^{\prime \prime} \times 0.4^{\prime \prime}$.

of the high-frequency spectrum and the high degree of symmetry of the source suggests that the compact central component could be identified to be a compact symmetric source (CSO) and this small size radio source is a restarted young source. We want also emphasize how rare it is to see extended emission in a CSO. Up to now the only known sources with these properties are 0108+388 (Stanghellini et al. 2005) and 0402+379 (Zhang et al. 2003). We have no optical spectroscopic information on this sources, so its classification is not possible.

\subsubsection{B2 $0722+30$}

This radio source is associated with a disk galaxy. Strong absorption is associated with its disk and a bulge-like component is also clearly visible. The radio emission originates from two symmetric lobe-like features in E-W direction, i.e. at an angle of $\sim 45^{\circ}$ to the disk (Capetti et al. 2000). In Fig. 7, we report the optical image from the HST (Hubble Space Telescope) overimposed on a VLA radio image at $5 \mathrm{GHz}$.

At arcsecond resolution, this source shows an FRI radio morphology with a total power of $3.1 \times 10^{23} \mathrm{~W} \mathrm{~Hz}^{-1}$ at $408 \mathrm{MHz}$ and a linear size of $\sim 13.5 \mathrm{kpc}$. 


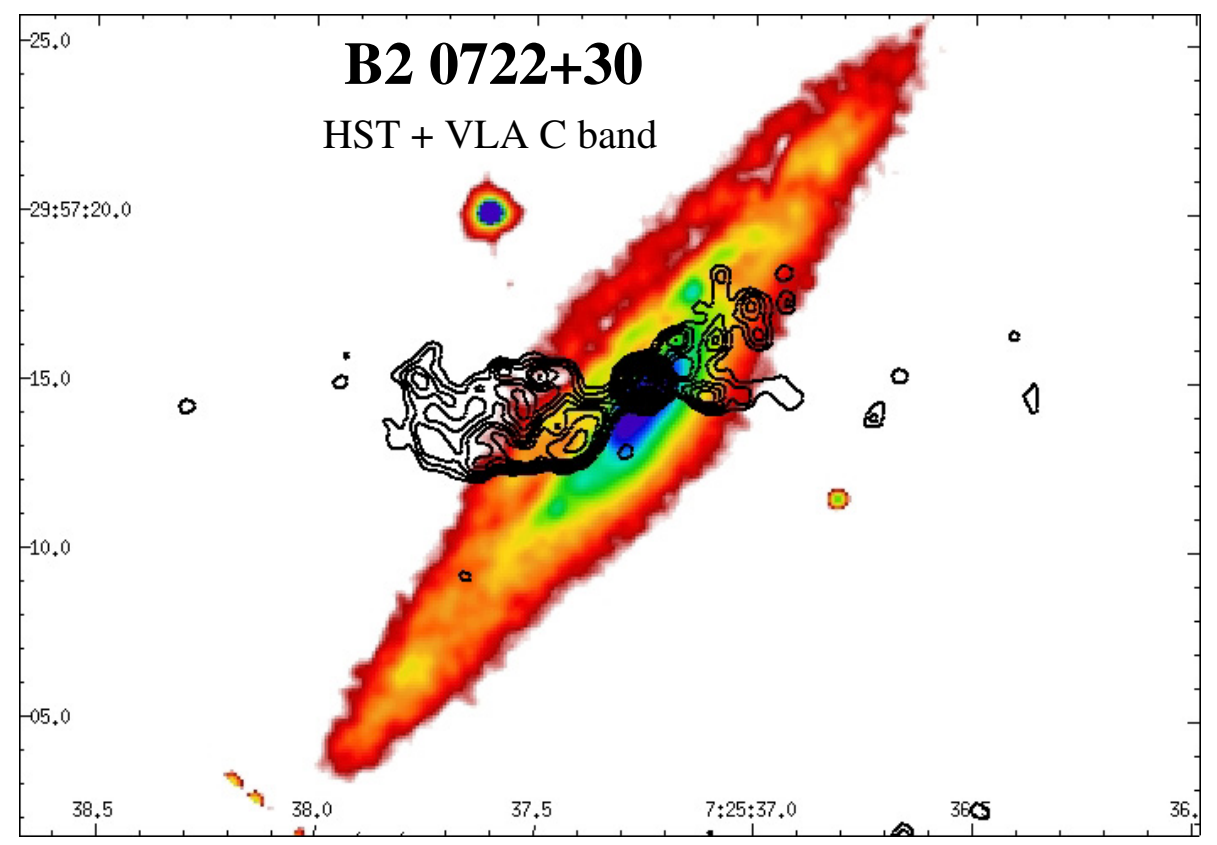

Fig. 7. VLA contour map in $C$ band of $\mathrm{B} 2$ $0722+30$ overimposed to HST image. Contour levels for the radio map are $0.15,0.20,0.25$, $0.30,0.35,0.4,0.5,0.7,1,2,3,5,7,9,12$, and $15 \mathrm{mJy} /$ beam. The beam size is $0.6^{\prime \prime} \times 0.6^{\prime \prime}, 0^{\circ}$. The peak level is $20.6 \mathrm{mJy} / \mathrm{beam}$.

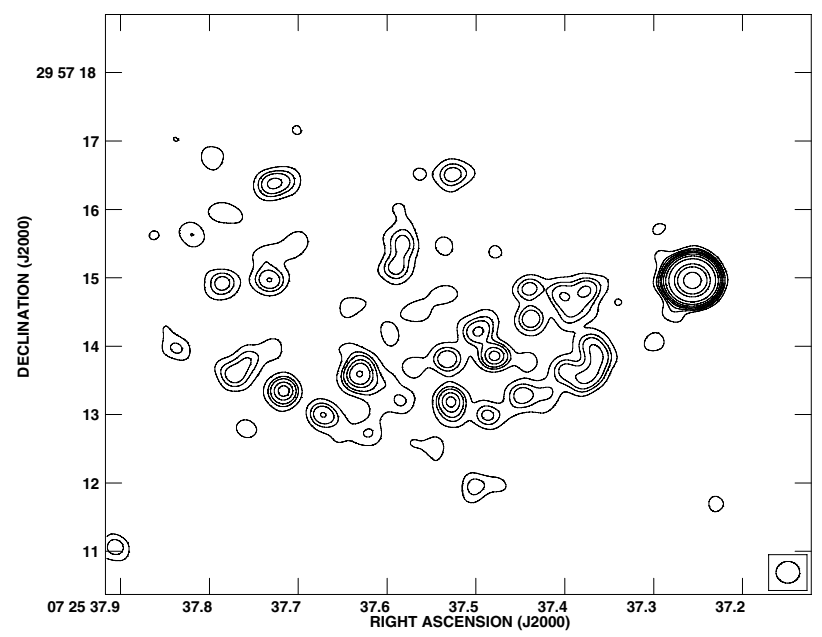

Fig. 8. 8.4 GHz VLA image of B2 $0722+30$. Contour levels are 0.035, $0.07,0.1,0.15,0.2,0.3,0.5,1,3,5,10$ and $15 \mathrm{mJy} / \mathrm{beam}$. The peak level is $14.6 \mathrm{mJy} / \mathrm{beam}$.

Our 8.4 GHz VLA observations (Fig. 8) show an unresolved nucleus and a marginal detection of the east lobe, which appears to be completely resolved. At $22 \mathrm{GHz}$, only the core is detected. It is unresolved with a total flux of $6.5 \mathrm{mJy}$ (Table 5). The radio core position is slightly offset with respect to the brightest optical region $\left(\sim 1^{\prime \prime}\right)$, but the optical core is only poorly defined because of the high dust absorption.

The core radio spectrum shows a clear steepening: $\alpha_{4.8}^{1.4}=$ $0.2, \alpha_{8.4}^{4.8}=0.64$, and $\alpha_{22}^{8.4}=0.85$ suggesting a sub-arcsecond resolved structure in agreement with the non detection in our VLBA data (Liuzzo et al. 2009b).

This source is one of the rare objects where a disk galaxy in the nearby Universe hosts a radio emission in FR I-like shape. Literature research can provide information on only three other cases: 1) the spiral galaxy 0313-192 in Abell 428 exhibiting a giant ( $350 \mathrm{kpc})$ double-lobed FRI (Ledlow et al. 1998, 2001; Keel et al. 2006); 2) NGC 612 is a typical S0 galaxy with a large-scale star-forming $\mathrm{H}$ I disk and a powerful FR-I/FR-II radio source (Véron-Cetty \& Veŕon 2001; Emonts et al. 2008); 3) the BL Lac object PKS 1413+135 (McHardy et al. 1994; Lamer et al. 1999).

In the optical, we found neither a spectrum, nor the [O III] emission line for this source: an optical classification is therefore not possible.

\subsubsection{B2 $1037+30$}

The source is only slightly resolved at $1.4 \mathrm{GHz}$ (Fanti et al. 1986). At subarsecond resolution at $8 \mathrm{GHz}$ we observe an edgebrightened structure with complex sub-structures: jets and lobes with hot spots are detected, while at $22 \mathrm{GHz}$ only a point-like component, probably the core, and the resolved NW hot spot are clearly visible. In VLBI images, the core is clearly revealed with a faint, diffuse emission detected on the shortest baselines (Giroletti et al. 2005b). According to its radio properties, Giroletti et al. (2005b) classified it as a young CSO source.

In the optical, B2 $1037+30$ is identified with the brightest galaxy in the cluster Abell 923. From the analysis, it follows that this is an LEG source: the optical line ratios set the source LEG region close to the boundary with HEG sources. The same position is found in the optical-extended radio plot, while in the optical-core radio plot and in the accretion rate plot the source in clearly identified with LEG types. The optical line ratios set the source close to the HEG-LEG boundary. The same position is found in the optical-radio plots.

\subsubsection{B2 $1101+38$}

Mrk 421 (B2 1101+38) is a well-known BL Lac, widely studied at all frequencies and detected at TeV energies (Punch et al. 1992). The NVSS (NRAO VLA Sky Survey) image shows a $30^{\prime \prime}$ core-dominated source, with emission on either side. The estimated viewing angle is $\theta \leq 20^{\circ}$. For more details on radio structure of this BL Lac, see Giroletti et al. (2006). 


\subsubsection{B2 1217+29}

This source is identified with the nearby galaxy NGC 4278. In the radio band it reveals a compact structure at all frequencies between 1.4 and $43 \mathrm{GHz}$ (Condon et al. 1998; Di Matteo et al. 2001; Nagar et al. 2000, 2001). Only at $8.4 \mathrm{GHz}$, at a resolution of $\sim 200$ mas, it is slightly resolved into a two-sided source with an extension to the south (Wilkinson et al. 1998). The VLBA data show a two-sided emission on sub-parsec scales in the form of twin jets emerging from a central compact component (Giroletti et al. 2005a). A large amount of an extended HI disk is detected in its central region (Emonts et al. 2006a,b).

This source shows a low $[\mathrm{O} \mathrm{III}] / \mathrm{H} \beta$ ratio, settling it in the LEG region of the diagnostic diagrams. In particular, it shows low [O III] luminosity, as confirmed by its position in the lower left side of the [O III]- radio plots.

\subsubsection{B2 1257+28}

This is one of two dominant members of the Coma cluster (Abell 1656) and is considered a BCG. It is a cD galaxy whit a small size WAT (wide angle tail) structure. Arcsecond-scale properties are discussed in Feretti \& Giovannini (1987): it has a total flux density at $1.4 \mathrm{GHz}$ of $190 \mathrm{mJy}$ and the core flux density at $6 \mathrm{~cm}$ is $1.1 \mathrm{mJy}$. The radio emission is completely embedded in the optical galaxy, and a gap of radio emission is present between the core and the SW lobe, while a faint jet connecting the core and the NE lobe is detected. Parsec-scale properties are discussed in detail in Liuzzo et al. (2010): the source shows a one-sided structure with a total flux density of $10.1 \mathrm{mJy}$, and the core flux density is $7.27 \mathrm{mJy}$.

Our optical analysis classified this source as an LEG-type object.

\subsubsection{C 272.1}

3C 272.1 is identified with a giant elliptical galaxy. It shows strong radio emission and a two-sided jet emerging from its compact core. HST images reveal dust lanes in the core of the galaxy while no significant amount of diffusely distributed cold dust was detected at sub-millimeter wavelengths (Leeuw et al. 2000). At parsec scale, this nearby source shows a clear onesided structure (Giovannini et al. 2001).

This source has a low [O III] luminosity, as confirmed by its position in the lower left side of the [O III]- radio plots.

\subsubsection{B2 1254+27}

This radio galaxy is identified with the BCG (brightest cluster galaxy) of a sub-group merging into the Coma cluster. The optical galaxy, NGC 4839, is classified as a cD galaxy (Parma et al. 1986, and references therein).

In VLA observations at $1.4 \mathrm{GHz}$, it appears as an FRI radio source with two lobes in NS direction. At $5 \mathrm{GHz}$, the arcsecond core flux density is $\sim 1.5 \mathrm{mJy}$ (Giovannini et al. 2005).

Our VLA maps at $8.4 \mathrm{GHz}$ and at $22 \mathrm{GHz}$ show a marginally resolved structure with an inverted spectral index (see Table 5), suggesting the presence of a compact nuclear emission.

The LEG classification of this source derived from the diagnostic diagrams is confirmed also by the [O III]-radio plots.

\subsubsection{B2 1322+36B}

On VLA scales, this source shows a twin-jet morphology. On parsec scale, it has a core-jet structure extending $\sim 10$ mas in the same direction as the main large-scale jet.

According to its position in all optical plots, this source is classified as an LEG.

\subsubsection{B2 1346+26}

This galaxy is the central galaxy of the cool core cluster A1795, which hosts the bright FR I radio source 4C 26.42. We studied its parsec-scale morphology in detail in Liuzzo et al. (2009a). Our multi-frequency and multi-epoch VLBA observations reveal a complex, reflection-symmetric morphology over a scale of a few mas. The source appears to be two-sided with a well-defined and symmetric Z-structure at $\sim 5$ mas from the core. The kiloparsecscale morphology is similar to the parsec-scale structure, but reversed in PA, with symmetric $90^{\circ}$ bends at about 2 arcsec from the nuclear region. A strong interaction with the ISM can explain the spectral index distribution and the presence of sub-relativistic jets on the parsec scale.

In the optical, even though we detect upper limits on the [O III] emission line, the position of this source in diagnostic diagrams reveals an LEG nature.

\subsubsection{C 305}

This small FR I radio galaxy shows a plumed double structure with two faint hot spots and symmetric jets. The optical galaxy is peculiar, with continuum emission on the HST scale perpendicular to the radio jet (Giovannini et al. 2005, and references therein). The X-ray emission of this source is extended but it is not connected with radio structure. However, the X-ray emission is cospatial with the optical emission line region dominated by the [O III]5007. This could be interpreted as due to the interaction between the radio jet and the ISM (Massaro et al. 2009).

From our optical analysis, according to its position in Fig. 3, this source is classified as HEG.

\subsubsection{B2 $1557+26$}

The host galaxy IC 4587 is a smooth and regular elliptical galaxy (Capetti et al. 2000).

In our $8.4 \mathrm{GHz}$ VLA observations (Fig. 9), the radiosource is resolved in a core and a NE jet aligned with the emission observed in the $5 \mathrm{GHz}$ VLBA images by Giovannini et al. (2005). The jet is extended about 0.6 arcsec from the core. At $22 \mathrm{GHz}$, the jet is not detected and the core has a total flux of $12.3 \mathrm{mJy}$ (Table 5).

According to its position in all the optical plots, this source is classified as LEG.

\subsubsection{B2 $1855+37$}

This source shows a distorted double structure on the kiloparsec scale, with no detection on VLBA scale, which suggests its identification with a small symmetric source with a faint core (Giovannini et al. 2005, and references therein). The extended structure of this source seems to be confined by external gas pressure.

Owing to upper limits on the optical emission lines, an optical classification is not possible. 


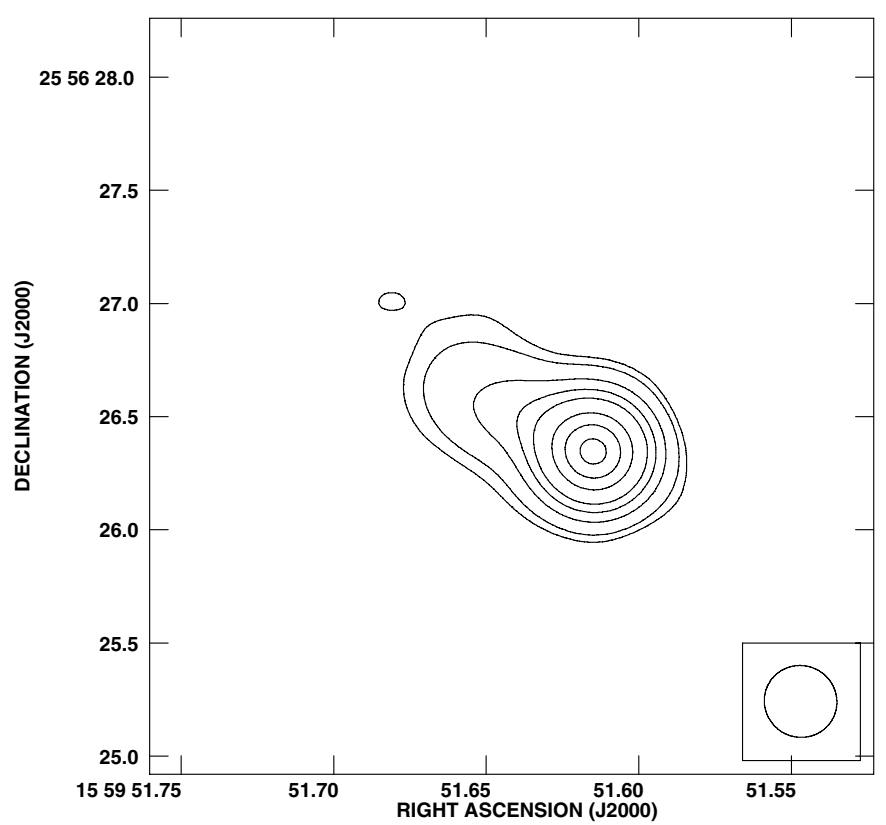

Fig. 9. 8.4 GHz VLA map of B2 1557+26. Contour levels are 0.15, 0.3, $0.9,1.8,3,6,9$, and $2 \mathrm{mJy} / \mathrm{beam}$. The peak level is $14.6 \mathrm{mJy} / \mathrm{beam}$.

\subsection{Extended C BCS sources}

\subsubsection{B2 $0331+39$}

Previous high-resolution VLA observations at $1.4 \mathrm{GHz}$ (A and B configuration) of this source (4C 39.12) revealed a resolved core plus a faint halo 1 arcmin in diameter (Parma et al. 1986; Fanti et al. 1986). Data at $5 \mathrm{GHz}$ also show that the core is resolved and the inner source region is dominated by a bright one-sided structure extended $\sim 1^{\prime \prime}$, and surrounded by a symmetric low-brightness halo.

This structure is confirmed by our $8.4 \mathrm{GHz}$ VLA image (Fig. 10): the radio source is characterized by a nuclear emission plus one-sided jet in the south direction and a halo around it. We note that the bright jet extension is not limited by sensitivity, but apparently the bright jet really ends after $\sim 1^{\prime \prime}$.

Our $22 \mathrm{GHz}$ VLA map (Fig. 10) shows an unresolved core and the southern jet. However, because of the high angular resolution and low surface brightness at this high frequency, the extended halo is not visible (see Table 5).

From Parma et al. (1986), we derived the spectral index between $1.4 \mathrm{GHz}$ and $8.4 \mathrm{GHz}$ of the halo: $\alpha_{8.4}^{1.4} \sim 0.4$. We also produced a spectral index image of the arcsecond jet comparing 8.4 and $22 \mathrm{GHz}$ images obtained using the same uv-range and angular resolution (Fig. 11). The core region has a flat spectral index with a clear steepening up to $\sim 0.9$ along the jet, which appears to be steeper than the surrounding halo.

The one-sided jet structure agrees well with previous VLBA observations of this source presented in Giovannini et al. (2001) where the parsec scale jet is found at the same PA of the arcsecond jet presented here.

At lower resolution this source is highly peculiar. From the NVSS image (see Fig. 10), we see a bright one-sided structure with a size of $\sim 3 \operatorname{arcmin}(70 \mathrm{kpc})$ oriented at $\mathrm{PA}=220^{\circ}$, i.e. very different from the jet and inner halo PA $\left(160^{\circ}\right)$. Moreover, a fainter symmetric diffuse emission is present on a larger angular scale (more than 6 arcmin, $150 \mathrm{kpc}$ ) at about the same PA of the inner one-sided jet and halo. The total NVSS flux density is $1.1 \mathrm{Jy}$. The total spectral index is $0.5-0.6$ from $74 \mathrm{MHz}$ up to
4.8 GHz (see NED archive data), with clear evidence that the newly restarted component is dominant at all frequencies.

This complex morphology suggests a restarting activity with a change of the PA in the different epochs. A more detailed study is necessary to understand this peculiar source.

We found neither the optical spectrum nor the [O III] emission line for this source: an optical classification is not possible.

\subsubsection{B2 $0844+31$}

This is a symmetric narrow-line FR II radio galaxy. At parsec resolution a bright core and two-sided jets are visible. The image from the FIRST Survey shows two extended FR I lobes at a larger distance from the core beyond the FR II-type hot spot, indicating a prior phase of radio activity. For this reason, this source could be classified as a restarted source, i.e., a source in which the extended FR I structure is related to previous activity, whereas the inner FR II structure originates from more recent core activity (Giovannini et al. 2005).

According to its position in all optical plots, this source is classified as an LEG.

\subsubsection{B2 $1003+35$}

It is the largest known FRII radio galaxy with a projected linear size of more than $6 \mathrm{Mpc}$. It shows a complex structure at arcsecond resolution with evidence of relativistic jets oriented in the same direction as the large-scale structure with some oscillations (Giovannini et al. 2005). Its peculiar radio structure has been interpreted as evidence of restarted activity (O'Dea et al. 2001).

According to its position in all optical plots, this source is classified as an LEG type, but close to the HEG boundaries.

\subsubsection{B2 $1144+35$}

This is a large-scale ( $\sim 0.9 \mathrm{Mpc})$ FRI radio source, coredominated with a short and bright two-sided jet. The bright arcsecond scale core is resolved at milliarcsecond resolution into a nuclear source, a main jet with an apparent superluminal velocity, and a faint counter-jet. There is evidence of a dynamic interaction with the surrounding medium. The radio morphology of this source shows clear discontinuities at different linear scales, suggesting a continuous activity but with high- and lowlevel periods (Giovannini et al. 2007).

From the optical point of view, this galaxy falls among the HEG sources in the diagnostic diagrams, showing a very strong [O III] $] \lambda$ 4959, $5007 \AA$ doublet. This classification is confirmed with the optical line luminosity - total and core radio power plots, as well as in the accretion rate plot.

\subsubsection{B2 $1512+30$}

The host galaxy does not show any outstanding morphological features, except for very faint elongated dust absorption at the center (Capetti et al. 2000). In the VLA image at $1.4 \mathrm{GHz}$ with 5 arcsec resolution, it appears as double source with two lobes in NS direction (Fanti et al. 1987), in agreement with the FIRST (Faint Images of Radio Sky at Twenty-Centimeters) image. The angular size is $\sim 22 \operatorname{arcsec}$ corresponding to $\sim 38 \mathrm{kpc}$.

Both in our $8.4 \mathrm{GHz}$ and $22 \mathrm{GHz}$ images, the source is undetected above $\geq 0.25 \mathrm{mJy} /$ beam and $\geq 0.30 \mathrm{mJy} /$ beam in $X$ - and $K$-bands respectively. 
E. Liuzzo et al.: Compact sources in the Bologna Complete Sample: high-resolution VLA observations and optical data
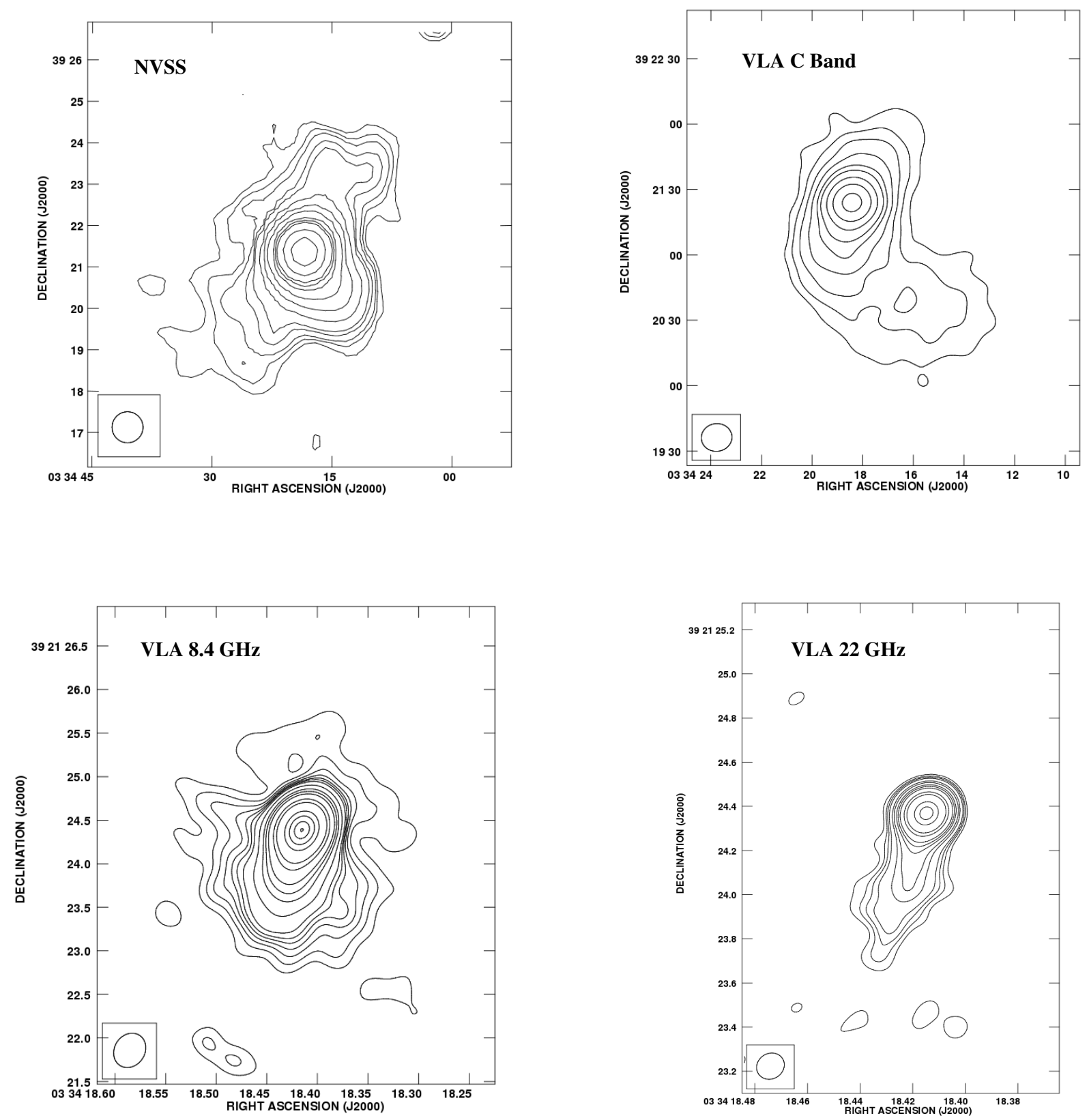

Fig. 10. Top left: NVSS image of B2 0331+39. Contour levels are 1, 1.5, 2, 3, 4, 8, 16, 32, 64, 90, 128, 256, and 512 mJy/b. Peak level is $811.2 \mathrm{mJy} /$ beam. Beam size is $45^{\prime \prime} \times 45^{\prime \prime}, 0^{\circ}$. Top right: VLA radio image in the $C$ Band of B2 0331. Contour levels are 1.6, 3.8, 7.8, 15.6, 31.1, $51.2,107.9,218$, and $436 \mathrm{mJy} / \mathrm{b}$. Peak level is $556.2 \mathrm{mJy} / \mathrm{beam}$. Beam size is $14^{\prime \prime} \times 12.7^{\prime \prime},-83^{\circ}$. Bottom left: $8.4 \mathrm{GHz}$ VLA image of B2 $0331+39$. Contour levels are $0.7,0.9,1.1,1.3,1.5,1.7,2,3,5,7,10,20,30,50,70,100,130$, and $150 \mathrm{mJy} / \mathrm{beam}$. The beam size is $0.41^{\prime \prime} \times 0.35^{\prime \prime}, \mathrm{PA}=-33$. The noise level is $0.1 \mathrm{mJy} /$ beam. Bottom right: $22 \mathrm{GHz}$ VLA image of B2 0331+39. Contour levels are 0.3, 0.5, 0.7, 1, 2, 3, 5, 7, 10, 20, 30, 50, 70 , and $100 \mathrm{mJy} /$ beam. The peak level is $117.1 \mathrm{mJy} /$ beam.

Using the total flux at $408 \mathrm{MHz}$ and at $1.4 \mathrm{GHz}$, we derived a spectral index $\alpha_{1.4}^{408 \mathrm{GHz}} \sim 2$. The non detection of a core emission at high frequency together with the steep low-frequency spectral index suggest that this object is a dying radiosource with a radio quiet core.

From our optical study, according to its position in the diagnostic diagrams (Fig. 3), this source is classified as an LEG.

\subsubsection{B2 $1626+39$}

This source is identified with the central galaxy 3C 338 in the cool core cluster A2199. It is a multiple-nuclei cD galaxy with dust lanes (Jensen et al. 2001). On kiloparsec scales it has two symmetric extended radio lobes that are characterized by a steep spectrum and are misaligned with the central emission. The two radio lobes are connected by a bright filamentary structure. Both the steep radio spectrum and strong filamentary emission may be caused by interactions with the dense intracluster medium (Gentile et al. 2007). 3C 338 was the first radio source in which a two-sided jet was observed on parsec scales (Feretti et al. 1993).

In the optical, according to its position in the optical-radio plots, this source is classified as an LEG source.

\section{Discussion}

We show in Fig. 12 a radio power vs. linear size diagram for the 95 sources of the whole BCS sample. Looking at the radio properties of C BCSs (red squares), some compact sources match 
Table 6. Properties of compact and extended BCS sources.

\begin{tabular}{|c|c|c|c|c|c|c|c|}
\hline Name & Morphology & $\begin{array}{c}\text { Optical } \\
\text { clas }\end{array}$ & $\begin{array}{l}\text { LLS } \\
\mathrm{kpc}\end{array}$ & $\begin{array}{c}\log P(408) \\
\mathrm{W} / \mathrm{Hz}\end{array}$ & $\begin{array}{c}\log L_{[\mathrm{OIII}]} \\
(\mathrm{erg} / \mathrm{s})\end{array}$ & Type & Notes \\
\hline B2 $0116+31$ & $\mathrm{CSO}$ & LEG & 0.1 & 25.46 & 40.39 & young & 4C31.04 \\
\hline B2 $0149+35$ & FR I & LEG & 18.8 & 23.32 & 39.10 & dying/recurrent & $\mathrm{BCG}$ in $\mathrm{A} 262$ \\
\hline B2 $0222+36$ & $\mathrm{C}$ & LEG & 4.5 & 23.93 & 40.71 & young/restarted & jet instability \\
\hline B2 $0258+35$ & CSS & LEG & 1.3 & 24.35 & 40.38 & HI-rich & frustrated \\
\hline B2 $0648+27$ & $\mathrm{C}$ & HEG & 1.2 & 24.02 & 41.58 & HI-rich & frustrated \\
\hline B2 $0708+32 B$ & FR I & ? & 10.2 & 24.49 & - & young & CSO -like \\
\hline B2 $0722+30$ & FR I & ? & 13.5 & 23.49 & - & peculiar & Disk galaxy \\
\hline B2 $1037+30$ & $\mathrm{CSO}$ & LEG & 5.0 & 25.36 & 40.08 & young & BCG in A923 \\
\hline B2 $1101+38$ & BL-Lac & $?$ & 18 & 24.39 & 40.54 & orientation & MKN421 \\
\hline B2 $1217+29$ & $\mathrm{C}$ & LEG & 0.01 & 21.75 & 38.83 & HI-rich & N4278 low power/frustrated \\
\hline $3 \mathrm{C} 272.1$ & FR I & LEG & 14.6 & 23.59 & 38.20 & young & \\
\hline B2 $1254+27$ & FR I & LEG & 16.7 & 23.45 & 39.01 & young & BCG group \\
\hline B2 $1257+28$ & FRI & LEG & 9.6 & 23.81 & 40.86 & frustrated & N4874, BCG in Coma \\
\hline B2 $1322+36 B$ & FR I & LEG & 18.7 & 24.07 & 39.42 & young & \\
\hline B2 $1346+26$ & FR I & LEG & 13.2 & 25.47 & 40.58 & frustrated/jet instability & BCG A1795 \\
\hline 3C 305 & FR I & HEG & 11.2 & 25.44 & 41.05 & frustrated & \\
\hline B2 $1557+26$ & $\mathrm{C}$ & LEG & 3.1 & 24.05 & 39.29 & low-power/frustrated & \\
\hline B2 $1855+37$ & $\mathrm{C}$ & LEG & 10.6 & 24.75 & & dying/restarted & \\
\hline B2 $0331+39$ & FRI & In & 70 & 24.19 & - & restarted & \\
\hline B2 $0844+31$ & FRII & LEG & 383.2 & 25.50 & 40.35 & restarted & IC 2402 \\
\hline B2 $1003+35$ & FRII & LEG & 4475 & 26.12 & 41.07 & restarted & $3 \mathrm{C} 286$ \\
\hline B2 $1144+35$ & FRI & HEG & 839 & 24.41 & 41.14 & high/low-phase & \\
\hline B2 $1512+30$ & FRI & LEG & 38 & 24.71 & 40.02 & dying & \\
\hline B2 $1626+39$ & FRI & LEG & 61.9 & 25.56 & 39.57 & & $3 \mathrm{C} 338, \mathrm{BCG}$ in $\mathrm{A} 2199$ \\
\hline
\end{tabular}

Notes. Column 1: names of the sources; Col. 2: radio morphologies; Col. 3: optical identification, where ? indicates source for which we could give a classification; Col. 4: linear size; Col. 5: logarithm of total radio power at $408 \mathrm{MHz}$ in units of W/Hz; Col. 6: logarithm of [OIII] $] 5007 \AA$ line luminosity in unit of $10^{-15} \mathrm{erg} \mathrm{s}^{-1}$; Col. 7: source classification based on our radio and optical analysis; Col. 8: notes on sources properties.

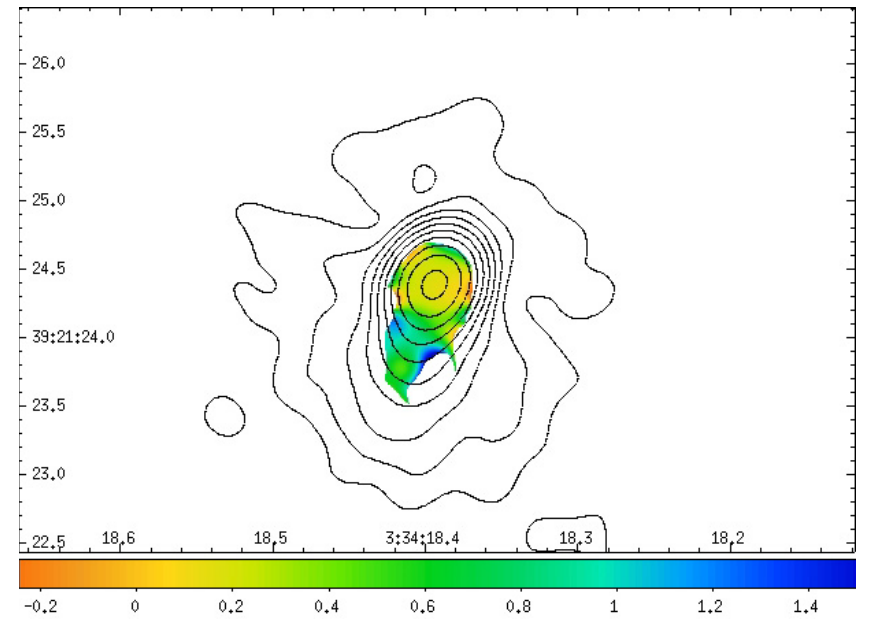

Fig. 11. Spectral index map (color) of B2 $0331+39$ between 8.4 and $22 \mathrm{GHz}$ with the $8.4 \mathrm{GHz}$ VLA emission (contour image) overimposed.

the general correlation between the linear size and the radio power, show a radio power at $408 \mathrm{MHz}$ lower than $10^{24} \mathrm{~W} / \mathrm{Hz}$. However, about half of the compact sources show a radio power higher than $10^{24} \mathrm{~W} / \mathrm{Hz}$.i.e., they are in the same range as classical extended radio galaxies.

To better investigate the nature and properties of C BCS properties, we compared optical and radio data. From Fig. 3, we note at first that the majority of C BCS sources are LEG, excepet for few. These peculiar compact sources are B2 0648+27 identified with a high rich HI galaxy (Emonts et al. 2006a,b), and $3 \mathrm{C} 305$, which is identified with a peculiar gas-rich galaxy

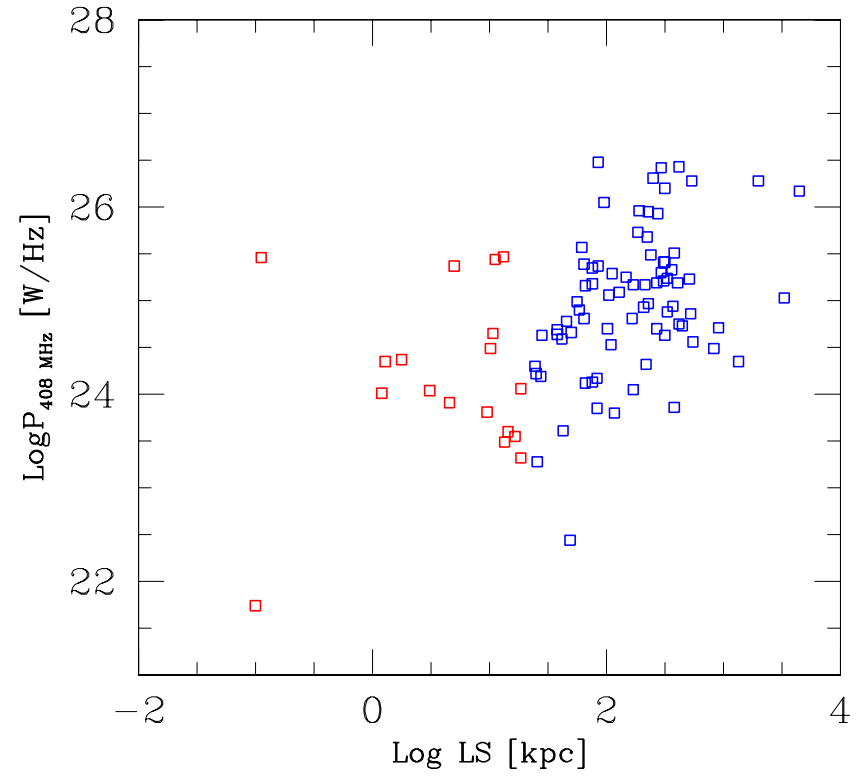

Fig. 12. Radio power vs. linear size diagram for sources in the BCS (Giovannini et al. 2005). The red squares indicate C BCS sources, while the blue ones represent the remaining extended objects.

discussed in detail by Massaro et al. (2009). Furthermore, one FR I BCS is among the HEG galaxies. This source, studied in detail by Giovannini et al. (2005), shows evidence of a multiphase radio activity. Recently, it restarted radio activity, and it shows structures on the parsec scale that move with an apparent velocity higher than $\mathrm{c}$. We conclude that for all three cases, 
the optical type can be explained by the peculiar activity of the source inner region (restarted activity or strong confinement and interaction with the surrounding medium).

In Fig. 4 we clearly see that $\mathrm{C}$ BCS sources show a correlation between total radio power and [OIII] luminosity in agreement with the core radio galaxies of Baldi \& Capetti (2010) and not with FR I radio galaxies. Furthermore, ther three HEG sources are in the same correlation, while the few other BCS FR I or FR II sources show the same correlation as 3C radio galaxies. These considerations seem to suggest that among LEG galaxies two different populations are present according to their radio and [OIII] luminosity: FR I radio galaxies and core radio galaxies $+\mathrm{C}$ BCS. In particular, we note that, at given total radio power, compact sources have an overluminous [O III] emission at a given radio power with respect to HEG and LEG 3CR sources. Moreover, if we consider the core radio power at $5 \mathrm{GHz}$, compact sources show a different trend with respect to LEG radio galaxies: they are overluminous in the optical at low radio power (CoRGs) and with properties similar to 3CR FRI at higher radio power (C BCS). Finally, the data suggest that CSS could follow the same trend as the compact sources, becoming optically underluminous at very high radio power. A larger statistics is necessary to clarify this point.

To better understand this correlation, we take the opportunity of the many available data for C BCS sources to investigate their origin.

Among C BCSs, we have quite different sources:

- A source can be compact because of projection effects: in our sample only one source is clearly oriented at a small angle with respect to the line of sight: B2 1101+38 (Mkn 421, Table 6). This source is a well- known BL-Lac type object (Giroletti et al. 2006) and its size is affected by strong projection effects. As discussed in Liuzzo et al. (2009b), the percentage of sources oriented at small angle with respect to the line of sight in the BCS agrees with unified models prediction.

- A source can be compact because it is young or has restarted activity: in our sample many sources show evidence of recent nuclear activity similar to CSO and CSS sources (e.g., B2 0258+35, Table 6), or restarted/recurrent activity (e.g., B2 $0149+35$, Table 6). These sources are expected to have a strong interaction with the dense inner ISM (see e.g., B2 1346+26, the BCG in A1795, Table 6). A peculiar source is B2 $1855+37$ which is characterized by a low no-jet emission and could be in the final stage of this scenario, and we do not know if it will die or will restart a new radio activity. If we compare these type of compact sources with the extended BCSs with evidence of a restarted (e.g., B2 0331+39, B2 $0844+31$, and B2 $1144+35$, Table 6) or dying activity (e.g., B2 1512+30, Table 6) we find no differences in their OIII luminosity and $408 \mathrm{MHz}$ total power.

We note also that on $18 \mathrm{C} \mathrm{BCSs,} \mathrm{six} \mathrm{sources} \mathrm{are} \mathrm{in} \mathrm{clus-}$ ters/groups; these are the BCGs. Literature studies (O'Dea et al. 2001) observed that compact, low-power and steepspectrum radio sources in BCGs are quite frequent. The radio properties of these objects could be explained if nuclear fueling is related to the AGN activity cycle, and we see here galaxies in a period of relative AGN quiescence or that are just restarted (young). The presence of restarted and cyclic radio activity in these clusters are requested by the cooling scenario (McNamara et al. 2005, and references therein). We claim that the fraction of $\mathrm{C} \mathrm{BCS}$ sources that are BGCs is considerable, corresponding, as discussed above, to $1 / 3(6 / 18)$ of the C BCSs. If we consider the whole BCS sample, the fraction of BGCs is $10 / 95$. The majority of the ten BCGs $(6 / 10)$ are compact BCS sources. This seems to suggest that the strong interaction with the dense ISM of the cluster environment increases the probability that the source is a compact radiosource, due to frustration effect and/or jet instability. However, optical properties of extended BCGs (see Table 6 and Sect. 5) and compact BCGs are similar.

- Sources in HI-rich galaxies: the results of Emonts et al. $(2006 a, b)$ revealed that FRI-type galaxies lie in a particular region of the HI mass disk/radio power diagram. However, there are sources that differ from FRI-type galaxies. They have a high value of HI mass. Studying these objects in detail, the authors found that they are compact sources, even if not all compact sources have a large HI mass. Some of our C BCS sources (B2 0648+27, B2 0258+35, and NGC 4278, Table 6) show a large amount of extended HI disk. For these HI-rich compact objects, Emonts et al. (2006a,b) suggested that they do not grow into extended sources because they are frustrated by the ISM in the central region of the galaxy, or because the fueling stops before the sources can expand. If these H I-rich radio galaxies formed through a major merger of gas-rich galaxies, part of the gas is expelled in largescale tidal structures, while another part is transported into the central kpc-scale region (e.g., Barnes 2002). The latter could be responsible for frustrating the radio jets if they are not too powerful. Alternatively, while the geometry and the conditions of the encounters appear to be able to form the observed large-scale HI structures, they might not be efficient in channeling substantial amounts of gas to the very inner pcscale region. This might prevent stable fueling of the AGN and hence large-scale radio structures do not develop. This hypothesis seems reasonable also considering our C BCSs: evidence that NGC 4278 and B2 0648+27 cannot grow as they are frustrated by the local ISM are present (Giroletti et al. 2005b); while B2 $0258+35$ displays variable levels of activity, suggestive of inefficient fueling, to expand beyond the kpc scale (Giroletti et al. 2005b).

We also found a slight correlation between the amount of HI mass and the central radio morphology: objects with high HI mass (e.g., B2 0258+35) show more diffuse radio emission in the central region.

Emonts et al. (2006a,b) discussed that HI-rich low-power compact sources have a different formation history from FRI objects which are likely the products of a major merger, as the detected large amounts of HI demonstrate. In this context, it is interesting that for B2 $0648+27$ and B2 1217+29 a major merger is clearly confirmed (Emonts et al. 2006a,b).

\section{Conclusion}

Radio galaxies are classified as FRI, FRII, and compact sources according to their powers and morphologies. Compact objects show emission properties that are not yet well understood. To investigate this peculiar class of sources, we selected from the Bologna Complete Sample (BCS, Giovannini et al. 2001, 2005; Liuzzo et al. 2009b) all objects with a linear size smaller than $20 \mathrm{kpc}$, which form the C BCS. Part of these targets were previously analyzed by us in the radio band (Giroletti et al. 2005b). Here, we completed the radio analysis of the C BCS sample, presenting new high-resolution VLA observations for the remaining sources. Moreover, we discussed for the first time all optical available data for C BCSs. 
From the comparison between $\mathrm{C}$ BCSs and other source samples/extended radiogalaxies, we derive that:

- Diagnostic diagrams reveal that with a few exceptions, C BCSs show similar optical LEG properties as the 3CR FR I radio galaxies and CoRGs of Baldi \& Capetti (2010).

- The optical [OIII] - radio correlations (total and nuclear radio power) suggest a common linear correlation for CoRG and $\mathrm{C}$ BCS sources, different from the known linear correlation of HEG and LEG 3CR radio galaxies, suggesting that $\mathrm{C}$ BCSs could be the powerful tail of CoRGs.

A possible continuity with powerful CSS it is not yet clear.

- According to our sub-arcsec radio data, the compactness of C BCSs is mostly caused by a young source age and/or restarted activity in a gas rich environment (e.g., BCG galaxies and HI-rich galaxies). Projection effects agree in some very few cases with unified model predictions.

Acknowledgements. This work was supported by contributions of European Union, Valle D'Aosta Region and the Italian Minister for Work and Welfare. This research has made use of the NASA/IPAC Extragalactic Data Base (NED), which is operated by the JPL, California Institute of Technology, under contract with the National Aeronautics and Space Administration.

\section{References}

Abazajian, K. N., Adelman-McCarthy, J. K., Agüeros, M. A., et al. 2009, ApJS, 182,543

Anton, K. 1993, A\&A, 270, 60

Baldi, R. D., \& Capetti, A. 2010, A\&A, 519, A48

Baldwin, J. A., Phillips, M. M., \& Terlevich, R. 1981, PASP, 93, 5

Barnes, J. E. 2002, MNRAS, 333, 481

Baum, S. A., \& Heckman, T. 1989a, ApJ, 336, 681

Baum, S. A., \& Heckman, T. 1989b, ApJ, 336, 702

Blanton, E. L., Sarazin, C. L., McNamara, B. R., \& Clarke, T. E. 2004, ApJ, 612, 817

Braine, J., \& Dupraz, C. 1994, A\&A, 283, 407

Buttiglione, S., Capetti, A., Celotti, A., et al. 2009, A\&A, 495, 1033

Buttiglione, S., Capetti, A., Celotti, A., et al. 2010, A\&A, 509, A6

Burstein, D., \& Heiles, C. 1982, AJ, 87, 1165

Burstein, D., \& Heiles, C. 1984, ApJS, 54, 33

Capetti, A., de Ruiter, H. R., Fanti, R., et al. 2000, A\&A, 362, 871

Capetti, A., Raiteri, C. M., \& Buttiglione, S. 2010, A\&A, 516, A59

Capetti, A., Buttiglione, S., Axon, D. J., et al. 2011, A\&A, 527, L2

Cardelli, J. A., Clayton, G. C., \& Mathis, J. S. 1989, ApJ, 345, 245

Condon, J. J., Yin, Q. F., Thuan, T. X., \& Boller, T. 1998, AJ, 116, 2682

Cotton, W. D., Feretti, L., Giovannini, G., et al. 1995, ApJ, 452, 605

Crawford, C. S., Allen, S. W., Ebeling, H., Edge, A. C., \& Fabian, A. C. 1999, MNRAS, 306, 857

Di Matteo, T., Carilli, C. L., \& Fabian, A. C. 2001, ApJ, 547, 731

Emonts, B. H. C., Morganti, R., Oosterloo, T. A., et al. 2006a, Astron. Nachr., 327,139

Emonts, B. H. C., Morganti, R., Tadhunter, C. N., et al. 2006b, A\&A, 454, 125

Emonts, B. H. C., Morganti, R., Oosterloo, T. A., et al. 2008, MNRAS, 387, 197

Fanaroff, B. L., \& Riley, J. M. 1974, MNRAS, 167, 31

Fanti, C., Fanti, R., de Ruiter, H. R., \& Parma, P. 1986, A\&AS, 65, 145
Fanti, C., Fanti, R., de Ruiter, H. R., \& Parma, P. 1987, A\&AS, 69, 57

Fanti, C., Fanti, R., Dallacasa, D., et al. 1995, A\&A, 302, 317

Feretti, L., \& Giovannini, G. 1987, A\&A, 182, 15

Feretti, L., Comoretto, G., Giovannini, G., Venturi, T., \& Wehrle, A. E. 1993, ApJ, 408, 446

Gelderman, R., \& Whittle, M. 1994, ApJS, 91, 491

Gentile, G., Rodríguez, C., Taylor, G. B., et al. 2007, ApJ, 659, 225

Giovannini, G., Cotton, W. D., Feretti, L., Lara, L., \& Venturi, T. 2001, ApJ, 552, 508

Giovannini, G., Taylor, G. B., Feretti, L., et al. 2005, ApJ, 618, 635

Giovannini, G., Giroletti, M., \& Taylor, G. B. 2007, A\&A, 474, 409

Giroletti, M., \& Panessa, F. 2009, ApJ, 706, L260

Giroletti, M., Giovannini, G., Taylor, G. B., et al. 2003, A\&A, 399, 889

Giroletti, M., Taylor, G. B., \& Giovannini, G. 2005a, ApJ, 622, 178

Giroletti, M., Giovannini, G., \& Taylor, G. B. 2005b, A\&A, 441, 89

Giroletti, M., Giovannini, G., Taylor, G. B., \& Falomo, R. 2006, ApJ, 646, 801

Heckman, T. M. 1980, A\&A, 87, 152

Ho, L. C., Filippenko, A. V., \& Sargent, W. L. W. 1997, ApJS, 112, 315

Jensen, J. B., Tonry, J. L., Thompson, R. I., et al. 2001, ApJ, 550, 503

Keel, W. C., White, R. E., III, Owen, F. N., \& Ledlow, M. J. 2006, AJ, 132, 2233

Kewley, L. J., Groves, B., Kauffmann, G., \& Heckman, T. 2006, MNRAS, 372, 961

Kunert-Bajraszewska, M., Gawroński, M. P., Labiano, A., \& Siemiginowska, A. 2010, MNRAS, 408, 2261

Laing, R. A., Riley, J. M., \& Longair, M. S. 1983, MNRAS, 204, 151

Lamer, G., Newsam, A. M., \& McHardy, I. M. 1999, MNRAS, 309, 1085

Ledlow, M. J., \& Owen, F. N. 1996, AJ, 112, 9

Ledlow, M. J., Owen, F. N., \& Keel, W. C. 1998, ApJ, 495, 227

Ledlow, M. J., Owen, F. N., Yun, M. S., \& Hill, J. M. 2001, ApJ, 552, 120

Leeuw, L. L., Sansom, A. E., \& Robson, E. I. 2000, MNRAS, 311, 683

Liuzzo, E., Taylor, G. B., Giovannini, G., \& Giroletti, M. 2009a, A\&A, 501, 933

Liuzzo, E., Giovannini, G., Giroletti, M., \& Taylor, G. B. 2009b, A\&A, 505, 509

Liuzzo, E., Giovannini, G., Giroletti, M., \& Taylor, G. B. 2010, A\&A, 516, A1

Massaro, F., Chiaberge, M., Grandi, P., et al. 2009, ApJ, 692, L123

McHardy, I. M., Merrifield, M. R., Abraham, R. G., \& Crawford, C. S. 1994, MNRAS, 268, 681

McNamara, B. R., Nulsen, P. E. J., Wise, M. W., et al. 2005, Nature, 433, 45

Morganti, R., Ulrich, M.-H., \& Tadhunter, C. N. 1992, MNRAS, 254, 546

Morganti, R., Tadhunter, C. N., Dickson, R., \& Shaw, M. 1997, A\&A, 326, 130

Morganti, R., Oosterloo, T. A., Capetti, A., et al. 2003, A\&A, 399, 511

Murgia, M., Fanti, C., Fanti, R., et al. 1999, A\&A, 345, 769

Murgia, M. 2003, PASA, 20, 19

Nagar, N. M., Falcke, H., Wilson, A. S., \& Ho, L. C. 2000, ApJ, 542, 186

Nagar, N. M., Wilson, A. S., \& Falcke, H. 2001, ApJ, 559, L87

O'Dea, C. P. 1998, PASP, 110, 493

O'Dea, C. P., Koekemoer, A. M., Baum, S. A., et al. 2001, AJ, 121, 1915

Parma, P., de Ruiter, H. R., Fanti, C., \& Fanti, R. 1986, A\&AS, 64, 135

Perlman, E. S., Stocke, J. T., Conway, J., \& Reynolds, C. 2001, AJ, 122, 536

Polatidis, A. G., \& Conway, J. E. 2003, PASA, 20, 69

Punch, M., Akerlof, C. W., Cawley, M. F., et al. 1992, Nature, 358, 477

Readhead, A. C. S., Pearson, T. J., Taylor, G. B., \& Wilkinson, P. N. 1996, Extragalactic Radio Sources, 175, 88

Sanghera, H. S., Saikia, D. J., Luedke, E., et al. 1995, A\&A, 295, 629

Stanghellini, C., O’Dea, C. P., Dallacasa, D., et al. 2005, A\&A, 443, 891

Urry, C. M., \& Padovani, P. 1995, PASP, 107, 803

Véron-Cetty, M. P., \& Véron, P. 2001, A\&A, 375, 791

van Breugel, W., Miley, G., \& Heckman, T. 1984, AJ, 89, 5

Wilkinson, P. N., Browne, I. W. A., Patnaik, A. R., Wrobel, J. M., \& Sorathia, B. 1998, MNRAS, 300, 790

Zhang, J.-S., Lin, R.-G., \& Fan, J.-H. 2003, Publications of the Yunnan Observatory, 95,98 